\title{
Properties and nature of Be stars
}

\section{Reliable physical properties of a semi-detached B9.5e+G8III binary BR CMi = HD 61273 compared to those of other well studied semi-detached emission-line binaries $\star, \star \star$}

\author{
P. Harmanec ${ }^{1}$, P. Koubský ${ }^{2}$, J. A. Nemravová ${ }^{1}$, F. Royer ${ }^{3}$, D. Briot ${ }^{3}$, P. North ${ }^{4}$, P. Lampens ${ }^{5}$, Y. Frémat ${ }^{5}$, S. Yang ${ }^{6}$, \\ H. Božić ${ }^{7}$, L. Kotková ${ }^{2}$, P. Škoda ${ }^{2}$, M. Šlechta ${ }^{2}$, D. Korčáková ${ }^{1}$, M. Wolf ${ }^{1}$, and P. Zasche ${ }^{1}$ \\ 1 Astronomical Institute of the Charles University, Faculty of Mathematics and Physics, V Holešovičkách 2, 18000 Praha 8 - Troja, \\ Czech Republic \\ e-mail: Petr.Harmanec@mff.cuni.cz \\ 2 Astronomical Institute, Academy of Sciences of the Czech Republic, 25165 Ondřejov, Czech Republic \\ 3 GEPI/CNRS UMR 8111, Observatoire de Paris, Université Paris Denis Diderot, 5 place Jules Janssen, 92910 Meudon, France \\ ${ }^{4}$ Laboratoire d'astrophysique, École Polytechnique Fédérale de Lausanne (EPFL), Observatoire de Sauverny, 1290 Versoix, \\ Switzerland \\ 5 Royal Observatory of Belgium, Ringlaan 3, 1180 Brussel, Belgium \\ 6 Physics \& Astronomy Department, University of Victoria, PO Box 3055 STN CSC, Victoria, BC, V8W 3P6, Canada \\ 7 Hvar Observatory, Faculty of Geodesy, University of Zagreb, Kačićeva 26, 10000 Zagreb, Croatia
}

Received 20 July 2014 / Accepted 25 October 2014

\section{ABSTRACT}

\begin{abstract}
Reliable determination of the basic physical properties of hot emission-line binaries with Roche-lobe filling secondaries is important for developing the theory of mass exchange in binaries. It is a very hard task, however, which is complicated by the presence of circumstellar matter in these systems. So far, only a small number of systems with accurate values of component masses, radii, and other properties are known. Here, we report the first detailed study of a new representative of this class of binaries, BR CMi, based on the analysis of radial velocities and multichannel photometry from several observatories, and compare its physical properties with those for other well-studied systems. BR CMi is an ellipsoidal variable seen under an intermediate orbital inclination of $\sim 51^{\circ}$, and it has an orbital period of 12 d 919059 (15) and a circular orbit. We used the disentangled component spectra to estimate the effective temperatures $9500(200) \mathrm{K}$ and 4655(50) $\mathrm{K}$ by comparing them with model spectra. They correspond to spectral types B9.5e and G8III. We also used the disentangled spectra of both binary components as templates for the 2D cross-correlation to obtain accurate radial velocities and a reliable orbital solution. Some evidence of a secular period increase at a rate of $(1.1 \pm 0.5) \mathrm{s}$ per year was found. This, together with a very low mass ratio of 0.06 and a normal mass and radius of the mass gaining component, indicates that BR CMi is in a slow phase of the mass exchange after the mass-ratio reversal. It thus belongs to a still poorly populated subgroup of Be stars for which the origin of Balmer emission lines is safely explained as a consequence of mass transfer between the binary components.
\end{abstract}

Key words. binaries: close - binaries: spectroscopic - stars: emission-line, Be - stars: individual: BR CMi (HD 61273) stars: fundamental parameters

\section{Introduction}

In spite of the concentrated effort of several generations of astronomers, the very nature of the Be phenomenon - the presence of the Balmer emission lines in the spectra of some stars

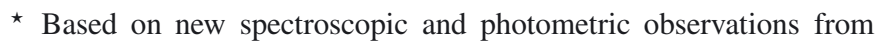
the following instruments: Elodie spectrograph of the Haute Provence Observatory, France; CCD coudé spectrograph of the Astronomical Institute AS ČR at Ondřejov, Czech Republic; CCD coudé spectrograph of the Dominion Astrophysical Observatory, Canada; HERMES spectrograph attached to the Mercator Telescope, operated on the island of La Palma by the Flemish Community, at the Spanish Observatorio del Roque de los Muchachos of the Instituto de Astrofísica de Canarias; 7-C photometer attached to the Mercator Telescope, La Palma, UBV photometers at Hvar and Sutherland, and $H_{\mathrm{p}}$ photometry from the ESA HIPPARCOS mission.

$\star \star$ Appendices are available in electronic form at http://www . aanda.org of spectral type B and their temporal variability on several time scales - is still not well understood. The competing hypotheses include (i) outflow of material from stellar photospheres, facilitated either by rotational instability at the stellar equator, by stellar wind, or by non-radial pulsations (or a combination of these effects); and (ii) several versions of mechanisms facilitated by the duplicity of the objects in question. It is true that the number of known binaries among Be stars is steadily increasing, but clear evidence that the Balmer emission is a consequence of the binary nature of the Be star in question exists only for Be stars, which have a mass-losing secondary that fills its Roche lobe. The number of known systems of this type with reliably determined physical properties is still rather small, and finding new representatives of this subgroup of Be binaries is desirable, not only for understanding Be stars but also from the point of view of the still developing theory of mass transfer in semi-detached binaries (see, e.g., the recent studies by Deschamps et al. 2013 or Davis et al. 2014). This paper deals with one such system. 
Table 1. Journal of RV data sets.

\begin{tabular}{ccrcc}
\hline \hline $\begin{array}{c}\text { Spg. } \\
\text { No. }\end{array}$ & Time interval & $\begin{array}{r}\text { No. } \\
\text { of } \\
\text { (HJD-2 450 000) }\end{array}$ & $\begin{array}{c}\text { WVavelength } \\
\text { range } \\
(\AA)\end{array}$ & $\begin{array}{c}\text { Spectral } \\
\text { res. }\end{array}$ \\
\hline 1 & $0071.5-3430.4$ & 34 & $3850-6800$ & 42000 \\
2 & $4000.6-5649.4$ & 56 & $6280-6730$ & 12700 \\
3 & $4733.0-5994.8$ & 14 & $6150-6760$ & 21700 \\
4 & $5278.4-5649.4$ & 19 & $8400-8900$ & 17000 \\
5 & $5589.6-5912.7$ & 8 & $3800-8750$ & $40000-90000$ \\
3 & $6031.7-6383.8$ & 5 & $6310-6920$ & 21700 \\
\hline
\end{tabular}

Notes. Column "Spectrograph No.": 1 OHP 1.93 m reflector, Elodie echelle spg.; 2 Ondřejov 2.0 m reflector, coudé grating spg., CCD detector, red spectra; 3 DAO 1.22 m reflector, coudé grating McKellar spg., CCD Site4 detector; 4 Ondřejov 2.0 m reflector, coudé grating spg., CCD detector, infrared spectra; 5 Mercator $1.2 \mathrm{~m}$ reflector, HERMES echelle spg.

BR CMi (HD 61273, BD+08¹831, SAO 115739, IRAS 07361+0804, HIP 37232) has not been studied much. Its light variability was discovered by Stetson (1991) from his uvby $\beta$ observations, but his individual observations have never been published. The variability was rediscovered by Perryman \& ESA (1997) with the HIPPARCOS satellite, and a period of 6.46 was reported by them. Kazarovets et al. (1999) classified it as a $\alpha^{2} \mathrm{CVn}$ variable and assigned it a variable-star name BR CMi. Paunzen et al. (2001) included this star into their list of $\lambda$ Boo candidates but conclude that it is a normal star of spectral type A6V. In preliminary studies, Briot \& Royer (2009) and Royer et al. (2007) announced that the object is actually a semi-detached Be dwarf and $\mathrm{K} 0$ giant binary and an ellipsoidal variable with an orbital period of 12.9190 and a mass ratio $M_{\mathrm{K}} / M_{\mathrm{B}}=0.14$. Hereafter, we call the Be star component 1 and the cool giant component 2. Renson \& Manfroid (2009) included the object into their catalogue of $\mathrm{Ap}, \mathrm{HgMn}$, and $\mathrm{Am}$ stars, giving it a spectral class A2, while Dubath et al. (2011), who were testing automatic classification procedures, classified it as an ellipsoidal variable with a period of 12.913 . We present the first detailed study of the system, based on a rich collection of spectral and photometric observations.

\section{Observations and reductions}

Spectroscopy: the star was observed at the Haute Provence (OHP), Ondřejov (OND), Dominion Astrophysical (DAO), and La Palma observatories. Table 1 gives a journal of all radial velocity (RV) observations. Details about the spectra reductions and RV measurements are given in Appendix A.

Photometry: we attempted to collect all available observations with known dates of observations. Basic information about all data sets can be found in Table 2 and the details on the photometric reductions and standardisation are in Appendix B. For convenience, we also publish all of our individual observations together with their HJDs there (in Tables B.3-B.6).

\section{Finding reliable orbital elements}

One always has to be cautious when analysing binaries with clear signatures of the presence of circumstellar matter in the system. The experience from other such systems shows that the $\mathrm{RV}$ curve of the Roche-lobe filling component is usually clean and defines its true orbit quite well, while the absorption lines of

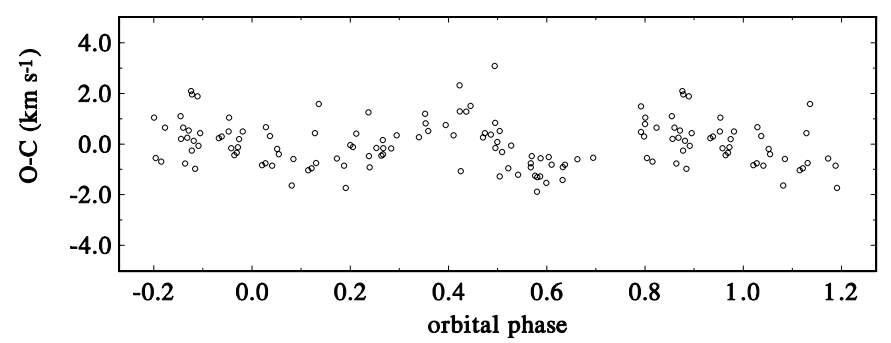

Fig. 1. O-C residuals from the circular-orbit solution 2 for all RVs of component 2 .

Table 2. Journal of available photometry for BR CMi.

\begin{tabular}{rcrcc}
\hline \hline Station & $\begin{array}{c}\text { Time interval } \\
\text { (HJD-2400 000) }\end{array}$ & $\begin{array}{r}\text { No. of } \\
\text { obs. }\end{array}$ & Passbands & Ref. \\
\hline 61 & $47912.6-49061.9$ & 111 & $V$ & 1 \\
37 & $52307.4-52384.4$ & 24 & $7-\mathrm{C}$ & 2 \\
93 & $52032.5-54836.8$ & 135 & $V$ & 3 \\
11 & $55567.4-55579.5$ & 19 & $U B V$ & 2 \\
01 & $55574.5-56015.3$ & 103 & $U B V$ & 2 \\
\hline
\end{tabular}

Notes. Individual observing stations are distinguished by the running numbers they have in the Prague/Zagreb photometric archives - see column "Station": 01 Hvar 0.65 m, Cassegrain reflector, EMI9789QB tube; 11 SAAO $0.5 \mathrm{~m}$ reflector, $U B V$ photometry; $37 \ldots$ Geneva $7-\mathrm{C}$ system: observations secured with the Mercator reflector at La Palma; 61 HIPPARCos all-sky $H_{\mathrm{p}}$ photometry transformed to Johnson $V$; 93 ASAS data archive (Pojmanski 2002).

Notes. Column "Ref.": 1) Perryman \& ESA (1997); 2) this paper; 3) Pojmanski (2002).

the mass-gaining component can be affected by the circumstellar matter, and their RV curves do not follow the true orbital motion (cf. Harmanec \& Scholz 1993; Desmet et al. 2010).

To derive an improved ephemeris and an orbital solution for component 2, we used all its RVs measured in SPEFO (see Appendix A for details) and the program FOTEL (Hadrava 1990, 2004a). These solutions are summarised in Table 3 . In solution 1 , individual systemic $(\gamma)$ velocities were derived for each spectrograph. Since our correction of the RV zero point via RV measurements of selected telluric lines clearly worked well (the $\gamma$ velocities of all four instruments being nearly identical), we assumed that all RVs are on the same system and derived solution 2 with a joint systemic velocity. In this solution, we weighted the individual datasets $1-4$ by weights inversely proportional to the squares of rms errors per one observation for each dataset from solution 1. As one can see in Fig. 1, the $\mathrm{O}-\mathrm{C}$ residuals from the circular-orbit solution 2 define a low-amplitude doublewave phase curve. For that reason, we also tentatively derived an eccentric-orbit solution. It indeed led to an eccentricity of $e=0.0085 \pm 0.0012$, which is statistically significant according to the test by Lucy \& Sweeney (1971). However, the corresponding longitude of periastron (referred to component 1) was $\omega=93.3 \pm 8.5$ degrees, which is suspect. It is known that for tidally distorted components of binaries, the difference between the optical and mass centre of gravity leads inevitably to a deformation of the RV curve, which manifests itself by a false eccentricity with values of $\omega$ either $90^{\circ}$ or $270^{\circ}$ (Sterne 1941; Harmanec 2003; Eaton 2008). This is what also occurs for BR CMi and similar binaries, e.g. AU Mon (Desmet et al. 2010) or V393 Sco (Mennickent et al. 2012b). 
P. Harmanec et al.: Duplicity of the Be star BR CMi

Table 3. Trial FOTEL orbital solutions based on RVs of component 2 measured in the program SPEFO.

\begin{tabular}{rcc}
\hline \hline Element & Solution 1 & Solution 2 \\
\hline$P(\mathrm{~d})$ & $12.919050 \pm 0.000021$ & $12.919046 \pm 0.000022$ \\
$T_{\text {super.c. }}$ & $54600.3786 \pm 0.0029$ & $54600.3806 \pm 0.0030$ \\
$e$ & 0.0 assumed & 0.0 assumed \\
$K_{2}\left(\mathrm{~km} \mathrm{~s}^{-1}\right)$ & $89.2086 \pm 0.0068$ & $89.1787 \pm 0.0070$ \\
$\gamma_{1}\left(\mathrm{~km} \mathrm{~s}^{-1}\right)$ & $-14.04 \pm 0.12$ & - \\
$\gamma_{2}\left(\mathrm{~km} \mathrm{~s}^{-1}\right)$ & $-14.12 \pm 0.15$ & - \\
$\gamma_{3}\left(\mathrm{~km} \mathrm{~s}^{-1}\right)$ & $-14.02 \pm 0.13$ & - \\
$\gamma_{5}\left(\mathrm{~km} \mathrm{~s}^{-1}\right)$ & $-14.49 \pm 0.28$ & - \\
$\gamma_{\text {joint }}\left(\mathrm{km} \mathrm{s}^{-1}\right)$ & - & $-14.088 \pm 0.088$ \\
$\mathrm{rms}\left(\mathrm{km} \mathrm{s}^{-1}\right)$ & 0.905 & 0.781 \\
No. of RVs & 117 & 117 \\
\hline
\end{tabular}

Notes. All epochs are in RJD = HJD-2 400 000; rms is the rms per 1 observation of unit weight. The systemic velocities $\gamma$ of individual spectrographs are identified by their numbers used in Table 1.

Consequently, we adopted the circular-orbit solution 2 in Table 3 as a basis for a working new ephemeris (1)

$$
T_{\text {super.c. }}=\text { HJD } 2454600.3806(30)+12.919046(22) \cdot E \text {. }
$$

Next we measured and analysed the $\mathrm{H} \alpha$ profile. Selected $\mathrm{H} \alpha$ profiles stacked with increasing orbital phase are shown in Fig. 2. For binary Be stars with strong $\mathrm{H} \alpha$ emission, this is the best measure of the true orbital motion of the Be component - see Božić et al. (1995), Ruždjak et al. (2009) and Peters et al. (2013). This is not so for BR CMi, since its $\mathrm{H} \alpha$ emission rises to only $20 \%$ above the continuum level, and the measured RV curve of the $\mathrm{H} \alpha$ emission wings is poorly defined and has a phase shift with respect to expected orbital motion of component 1. In Fig. 2 one can also note that the width of both peaks of the double $\mathrm{H} \alpha$ emission varies systematically with the orbital phase, being largest shortly after both conjunctions. Figure 2 also shows the secular stability of the strength of $\mathrm{H} \alpha$ emission, with the observed changes mainly due to RV shifts of two absorption components, the stronger one associated with component 2 and a weaker one associated with material in the neighbourhood of component 1. It exhibits a RV curve, which is not in exact antiphase to that of component 2 .

In another attempt we used the program KOREL ${ }^{1}$ developed by Hadrava $(1995,2004 b)$ for spectra disentangling. Since the expected semi-amplitude of the orbital RV curve of component 1 is low, we decided to only analyse high-resolution Elodie and Hermes spectra from the blue spectral region between 4397 and $4608 \AA$. This region contains a number of He I and metallic lines but no Balmer line (to avoid contamination by the circumstellar matter). It turned out that because of relatively broad and not very numerous line profiles of component 1 and a low semiamplitude of its RV curve, comparably good solutions could be obtained for a wide range of mass ratios. We note, however, that for the same reasons the disentangling of individual spectra is robust and can be trusted.

We then decided to try a cross-correlation in two dimensions, suggested (and realized as the program TODCOR) by Zucker \& Mazeh (1994). In this technique, the individual observed spectra are compared to the two template spectra, which should closely correspond to the spectra of the two binary components, both in the spectral type and the projected rotational broadening. The

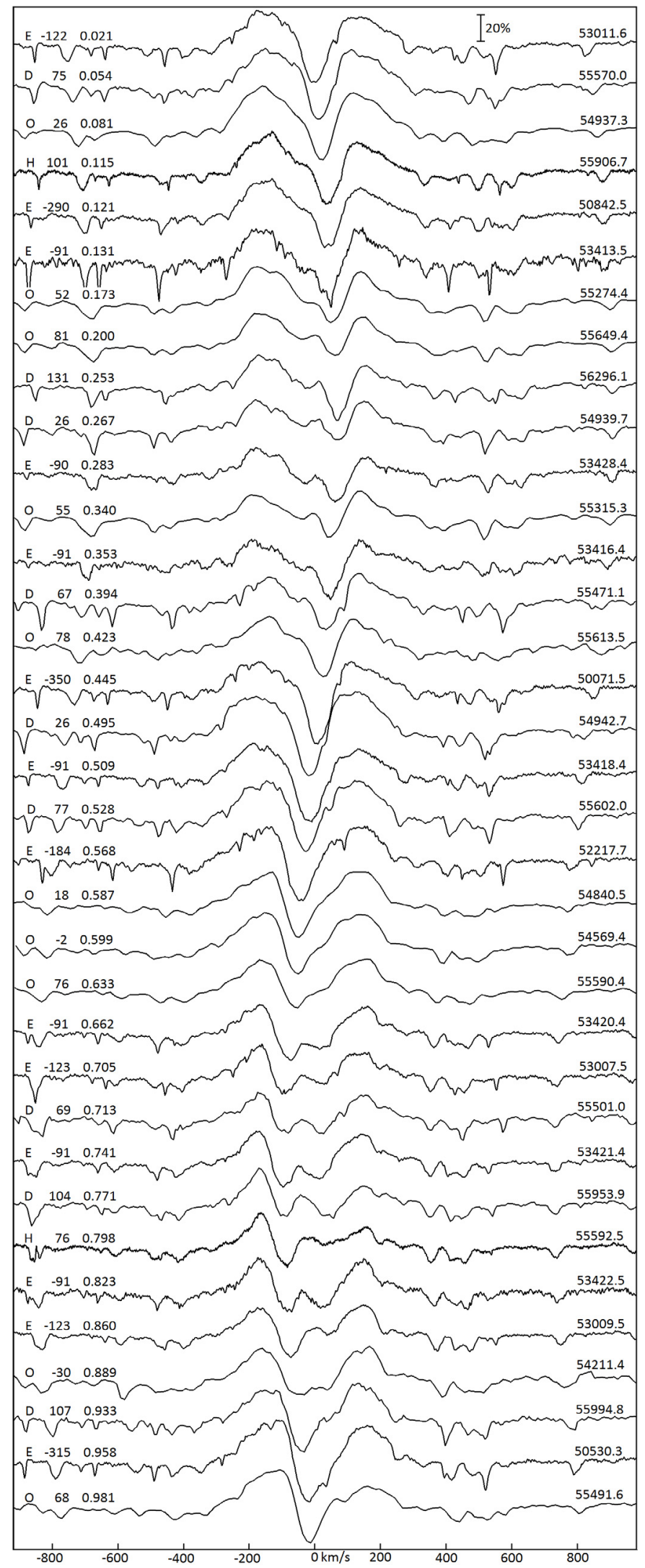

Fig. 2. Selected $\mathrm{H} \alpha$ line profiles of BR CMi stacked with increasing orbital phase. Profiles are identified by cycle numbers and orbital phases with respect to ephemeris (1) and by RJDs = HJDs -2400 000.0. Symbols E, O, D, and $\mathrm{H}$ stand for Elodie, Ondřejov, DAO, and Hermes spectrographs.

\footnotetext{
The freely distributed version from Dec. 2004.
} 


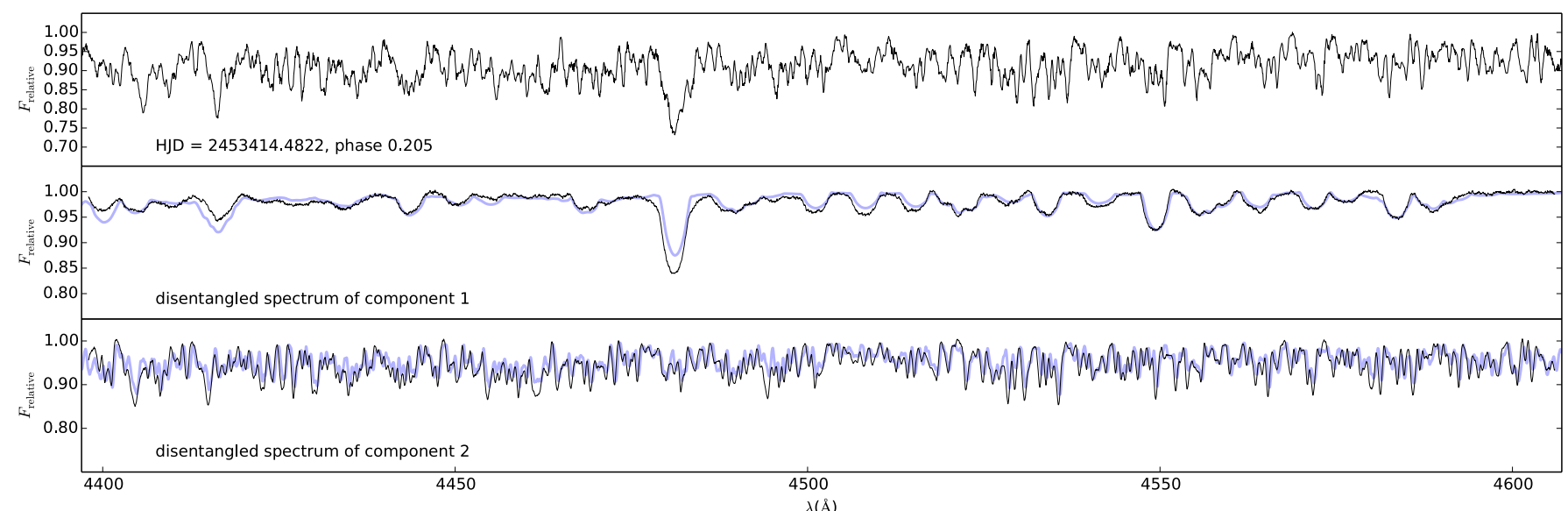

Fig. 3. One observed Elodie spectrum and the disentangled spectra of components 1 and 2 in the wavelength interval $4397-4608 \AA$. The disentangled spectra were renormalised to their own continua and are compared to a model fit (see Sect. 4.1).

RVs for both binary components are derived for each observed spectrum. A similar program to TODCOR was written by one of us (YF) under the name asTODCOR and has already been successfully applied to the case of AU Mon (Desmet et al. 2010). In practice, the Fourier transform of the observed and template spectra is computed via the FFT technique (Press et al. 1993). To meet the requirements of the FFT algorithm (see e.g. David et al. 2014), the spectra were rebinned to a smaller, constant, $\log \lambda$ bin size and apodised at both edges by means of a cosine-bell function. David \& Verschueren (1995) showed that the combination of an even and uneven number of points correctly chosen around the CCF summit allows reducing the impact of discretisation on the measurement of its location, hence on the RV determinations. This approach was therefore also implemented in asTODCOR by combining the results of a parabola fit through three and four points.

It is usual practice to use suitable synthetic and properly rotationally broadened spectra as the templates. In this case, we used the spectra of both components disentangled by KOREL as the templates, adopting a preliminary estimated luminosity ratio $L_{2} / L_{1}=0.25$. The wavelength interval 4397-4608 $\AA$ was used (see Fig. 3), and we verified that the disentangled spectrum of component 1 had the same systemic velocity as component 2 . The procedure worked remarkably well and returned well-defined RV curves for both binary components. This is seen in Fig. 4, which is the phase plot of these curves for ephemeris (1). We publish all individual SPEFO RVs for component 2 and asTODCOR RVs for both components, together with their HJDs in Tables A.2 and A.3.

In Table 4 we provide the circular-orbit solutions based on RVs resulting from 2D cross-correlation. Solution 3 is based on RVs of component 1 and solution 4 on RVs of component 2 alone. Their comparison shows that the epochs of superior conjunction of component 1 from both solutions agree with each other within the limits of their respective errors. They also agree with the epoch derived from a more numerous set of RVs from the red spectral region measured in SPEFO (see solution 2 of Table 3). Since the 2D cross-correlation puts no constraint on the derived RVs, this seems to prove that the RVs of component 1 , found to be in exact antiphase to those of component 2 , indeed reflect the true orbital motion of component 1 . In addition, both solutions have identical RV zero points within its rms error of $0.1 \mathrm{~km} \mathrm{~s}^{-1}$, confirming that using disentangled spectra has not introduced any biases. We therefore derived another solution 5 ,

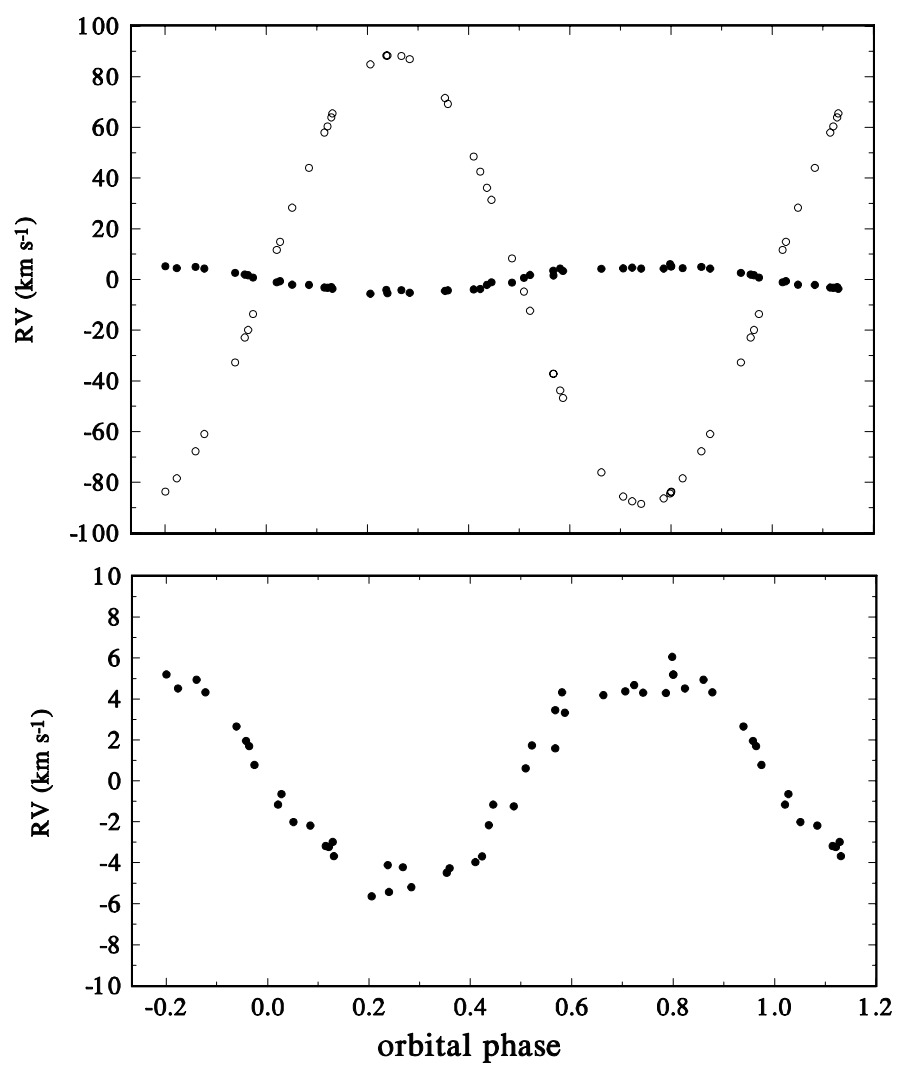

Fig. 4. Top: orbital RV curve of both components of BR CMi based on RVs derived via 2D cross-correlation. Bottom: zoomed RV curve of component 1 from the upper panel showing how accurately the RVs were derived via the $2 \mathrm{D}$ cross-correlation.

which is based on RVs for both binary components. It defines the $M_{1,2} \sin ^{3} i$ values and the mass ratio $M_{2} / M_{1}=0.0593$.

\section{An estimate of basic physical elements of the binary components and the whole system}

Since BR CMi is not an eclipsing but an ellipsoidal binary, its light curves can almost equally well be fitted for a wide range of possible orbital inclinations. For that reason, we proceeded in an iterative way, trying to describe all system properties in a mutually consistent way and taking all limitations we have at 
Table 4. FOTEL circular-orbit solutions individually for components 1 and 2 and for both stars together, based on RVs obtained via a 2D crosscorrelation.

\begin{tabular}{rrrr}
\hline \hline Element & Solution 3 & Solution 4 & Solution 5 \\
\hline$T_{\text {sup. conj. }}$ & 0.427 & 0.3807 & 0.3808 \\
& \pm 0.059 & \pm 0.0032 & \pm 0.0033 \\
$K_{1}\left(\mathrm{~km} \mathrm{~s}^{-1}\right)$ & $5.26 \pm 0.15$ & - & $5.26 \pm 0.14$ \\
$K_{2}\left(\mathrm{~km} \mathrm{~s}^{-1}\right)$ & - & $88.71 \pm 0.14$ & $88.71 \pm 0.15$ \\
$\mathrm{rms}\left(\mathrm{km} \mathrm{s}^{-1}\right)$ & 0.694 & 0.630 & 0.666 \\
No. of RVs & 41 & 41 & $41 / 41$ \\
\hline
\end{tabular}

Notes. The orbital period was fixed at 12 d 919046 in all cases. All epochs are in HJD-2 454600 ; rms is the rms of 1 observation of unit weight.

our disposal into account. In particular, we used the disentangled spectra of both binary components in several spectral regions to obtain good estimates of the effective temperatures of both stars via a comparison of disentangled and synthetic line profiles. These were then kept fixed in the combined light-curve and orbital solutions for a given inclination. Since the masses and radii thus obtained define $\log g$ quite accurately, we kept them fixed in the determination of $T_{\text {eff }}$.

\subsection{Comparing the disentangled and interpolated model spectra}

We used a program of J.A. Nemravová (see Nasseri et al. 2014) that interpolates in a precalculated grid of synthetic stellar spectra sampled in the effective temperature, gravitational acceleration, and metallicity. The synthetic spectra are compared to the disentangled ones for two or more components of a multiple system (normalised to the joint continuum of all bodies) and the initial parameters are optimised by minimisation of $\chi^{2}$ until the best match is achieved.

Since the fractional luminosities within a considered spectral region are treated as independent of wavelength, we fitted the following six disentangled spectral bands separately: 4002-4039, 4142-4191, 4381-4435, 4446-4561, 4910-4960, and 8400-8880 $\AA$. The interpolation was carried out in two different grids of spectra: AMBRE grid computed by de Laverny et al. (2012) was used for component 2, and the Pollux database computed by Palacios et al. (2010) was used for the hotter component 1 .

It would not be wise to derive all physical parameters via free convergence in all considered spectral regions. Since the combined light and orbital solution can provide very tight values of $\log g$ and systemic velocity $\gamma$, we kept these values fixed in each superiteration between model spectra and combined solution. The infrared region $8400-8880 \AA$ is the most appropriate region for the $T_{\text {eff }}$ determination of component 2 , since its fractional luminosity is much higher there $(\sim 0.5$ compared to less than 0.2 in the blue regions) and its spectrum is rich there.

Having the effective temperature of component 2 determined with an accuracy of $\sim 50 \mathrm{~K}$, we kept it fixed in all five blue spectral regions, where the fractional luminosity of component 2 is low. The uncertainty of the fitted parameters has two components. The first is the statistical one connected with the photon noise and represents a minor contribution since the disentangled profiles have very high $\mathrm{S} / \mathrm{N}$. The second is influenced by the approach to the minimisation and can be estimated by repeating the fitting procedure and the need for (re)normalisation of the
Table 5. Average results of the final best fit of the interpolated grid of synthetic spectra to the observed ones in the six considered spectral regions.

\begin{tabular}{lc|c}
\hline \hline Quantity & Component 1 & Component 2 \\
\hline$T_{\text {eff }}(\mathrm{K})$ & $9514 \pm 171$ & $4655 \pm 50$ \\
$v \sin i\left(\mathrm{~km} \mathrm{~s}^{-1}\right)$ & $130.98 \pm 3.27$ & $20.11 \pm 2.71$ \\
\hline
\end{tabular}

Notes. The majority of the physical properties affecting the line profiles were kept fixed from the final combined orbital and light-curve solution. $T_{\text {eff }}$ of component 2 is based on the fit in the IR region alone - see the text for details.

disentangled profiles ${ }^{2}$. The former source of systematic error is not taken into account, because our program does not normalise the disentangled spectra. Therefore, we estimated the uncertainties of the optimised parameters as a mean of the best fit values obtained for each modelled spectral band, which should (at least to some degree) account for the need for (re)normalisation of the disentangled profile. The only exception is the uncertainty of the effective temperature of component 2 , which was estimated via a simple Monte Carlo simulation. This is justified since the disentangled continua of component 2 were very close to a straight line in all regions. The results of the final minimisation averaged over all considered spectral regions are summarised in Table 5.

\subsection{A combined orbital and light-curve solution}

We used the program PHOEBE (version 1.0 with a few custom passbands added; Prša \& Zwitter 2005, 2006), all photometric data sets available to us, RVs of both components from the 2D cross-correlation and also RVs of component 2 measured in SPEFO because they give almost the same RV curve as the RVs from 2D cross-correlation but represent a much more numerous data set. For all Elodie and Hermes spectra, for which both sets of RVs of component 2 exist, we set their weights to 0.5 in both subsets. We assumed that component 2 fills the Roche lobe, fixed the mass ratio $M_{2} / M_{1}=0.0593$, which follows from the orbital solution for the cross-correlated RVs from spectra with high $\mathrm{S} / \mathrm{N}$ (see solution 5 of Table 4).

An unsuccessful attempt to converge $T_{\text {eff }}$ of component 1 was carried out. Changes of the $T_{\text {eff }}$ did not lead to any improvement in the cost function value, and the program merely adjusted the radii of both components to adapt the model to the new temperature. We therefore decided to fix the effective temperatures of both components obtained from the fit of synthetic spectra to the disentangled ones. We adopted $T_{\text {eff }}=4655 \mathrm{~K}$ for component 2 . The effective temperature of component 1 is rather uncertain. We therefore computed three sets of trial solutions for three different $T_{\text {eff }}$ of the component 1 corresponding to the estimated $1 \sigma$ bars given in Table 5: $T_{\text {eff }} \epsilon\{9343,9514,9685\} \mathrm{K}$. The solution that has the least $\chi^{2}$ of all trial solutions is listed in Table 6 . Error bars presented in Table 6 were estimated as the difference between the maximal (minimal) result of each parameter, which did not have the $\chi^{2}$ greater than $1 \%$ of the $\chi^{2}$ of the best solution, and the best solution. The bolometric albedos were estimated for the corresponding effective temperatures from Fig. 7 of Claret (2001) and the coefficients of gravity brightening from Fig. 7 of Claret (1998). Logarithmic limb-darkening coefficients were

2 Since the disentangling in KOREL is carried out in Fourier space, it
happens in almost every practical application that the resulting disentan-
gled spectra after inverse Fourier transformation have slightly warped continua and need some re-normalisation. 
Table 6. Combined RV curve and light-curve solution.

\begin{tabular}{|c|c|c|}
\hline Element & \multicolumn{2}{|c|}{ Orbital properties } \\
\hline $\begin{array}{l}P(\mathrm{~d}) \\
T_{\min }(\mathrm{RJD}) \\
M_{2} / M_{1} \\
i(\mathrm{deg}) \\
a\left(R_{\odot}\right)\end{array}$ & $\begin{array}{r}12.9190 \\
54600.38 \\
0.0 \\
50.8 \\
31.2 \\
\end{array}$ & $\begin{array}{l}9 \pm_{0.000015}^{0.000015} \\
86 \pm_{0.00206}^{0.0006} \\
93^{*} \\
\pm_{2.70}^{5.33} \\
\pm_{2.08}^{1.28} \\
\end{array}$ \\
\hline & \multicolumn{2}{|c|}{ Component properties } \\
\hline & Component 1 & Component 2 \\
\hline$T_{\text {eff }}(\mathrm{K})$ & $9685^{*}$ & $4655^{*}$ \\
\hline$\Omega$ & $277.2 \pm \pm_{26.8}^{5.0}$ & $22.82^{*}$ \\
\hline $\log g_{[\operatorname{cgs}]}$ & $4.228 \pm_{0.118}^{0.031}$ & $2.095 \pm_{0.033}^{0.018}$ \\
\hline$M\left(M_{\odot}\right)$ & $2.31 \pm_{0.43}^{0.29}$ & $0.137 \pm_{0.026}^{0.017}$ \\
\hline$R\left(R_{\odot}\right)$ & $1.93 \pm_{0.00}^{0.13}$ & $5.49 \pm_{0.37}^{0.22}$ \\
\hline$F$ & $22.40^{* *}$ & $1.00^{* *}$ \\
\hline$M_{\mathrm{bol}}$ & $1.080 \pm_{0.066}^{0.101}$ & $1.991 \pm_{0.085}^{0.152}$ \\
\hline$L_{\text {Johnson- } U}$ & $0.953 \pm_{0.009}^{0.008}$ & $0.047 \pm_{0.008}^{0.009}$ \\
\hline$L_{\text {Johnson }-B}$ & $0.890 \pm_{0.014}^{0.018}$ & $0.110 \pm_{0.018}^{0.0014}$ \\
\hline$L_{\text {Johnson- } V}$ & $0.740 \pm_{0.022}^{0.035}$ & $0.260 \pm_{0.035}^{0.022}$ \\
\hline$L_{\text {Geneva }-U}$ & $0.994 \pm_{0.001}^{0.001}$ & $0.006 \pm_{0.001}^{0.001}$ \\
\hline$L_{\text {Geneva }-B 1}$ & $0.984 \pm_{0.003}^{0.003}$ & $0.016 \pm_{0.003}^{0.003}$ \\
\hline$L_{\text {Geneva-B }}$ & $0.963 \pm_{0.006}^{0.007}$ & $0.037 \pm_{0.007}^{0.006}$ \\
\hline$L_{\text {Geneva- } B 2}$ & $0.936 \pm_{0.009}^{0.011}$ & $0.064 \pm_{0.011}^{0.009}$ \\
\hline$L_{\text {Geneva-V1 }}$ & $0.816 \pm_{0.018}^{0.027}$ & $0.184 \pm_{0.027}^{0.018}$ \\
\hline$L_{\text {Geneva- } V}$ & $0.800 \pm_{0.019}^{0.029}$ & $0.200 \pm_{0.029}^{0.019}$ \\
\hline$L_{\text {Geneva-G }}$ & $0.739 \pm_{0.021}^{0.036}$ & $0.261 \pm_{0.036}^{0.021}$ \\
\hline$L_{\mathrm{Hp}}$ & $0.804 \pm_{0.022}^{0.021}$ & $0.196 \pm_{0.021}^{0.021}$ \\
\hline
\end{tabular}

Notes. The optimised parameters are given with only their formal errors. ${ }^{(*)}$ Parameters that were fixed during the optimisation. ${ }^{(*)}$ The synchronicity parameter $F$ of component 1 was kept fixed during each iteration, but it was recalculated for new radius and inclination after each iteration, using $v \sin i$ from Table 5. A spin-orbit synchronization was assumed for the Roche lobe-filling component 2.

automatically interpolated after each iteration in PHOEBE from a pre-calculated grid of model atmospheres.

A test of the robustness of the final solution presented in Table 6 was carried out using the scripter environment of PHOEBE. We fitted the photometric observations and RVs from the $2 \mathrm{D}$ cross-correlation starting the convergence for 29 different initial orbital inclinations $i$ ranging from 30 to 86 degrees. During each run, 30 iterations were computed, although the solution usually converged in less than ten iterations. The rotation parameter $F$ (a ratio of spin to orbital revolution) of component 1 was not converged in PHOEBE, but it was optimised after each iteration using the new values of the component 1 radius and of the orbital inclination, while keeping the value of $v \sin i$ fixed from Table 5. A comparison of the optimal PHOEBE model light curves with the observed ones is in Fig. 5.

The free convergence of the orbital period and epoch, now based not only on RVs but also on photometry, led to an improved linear ephemeris (2)

$T_{\text {super.c. }}=$ HJD $2454600.38187(42)+12$ d.9190594(7) $\cdot E$.

To check on a secular constancy of the orbital period, we split the RVs and photometry into two subsets: HJD $2448705-$ 53650 containing 460 data, and HJD 24540 00-56295 containing 505 data. This particular choice ensured that both subsets contain a representative mixture of RVs and photometry. Local

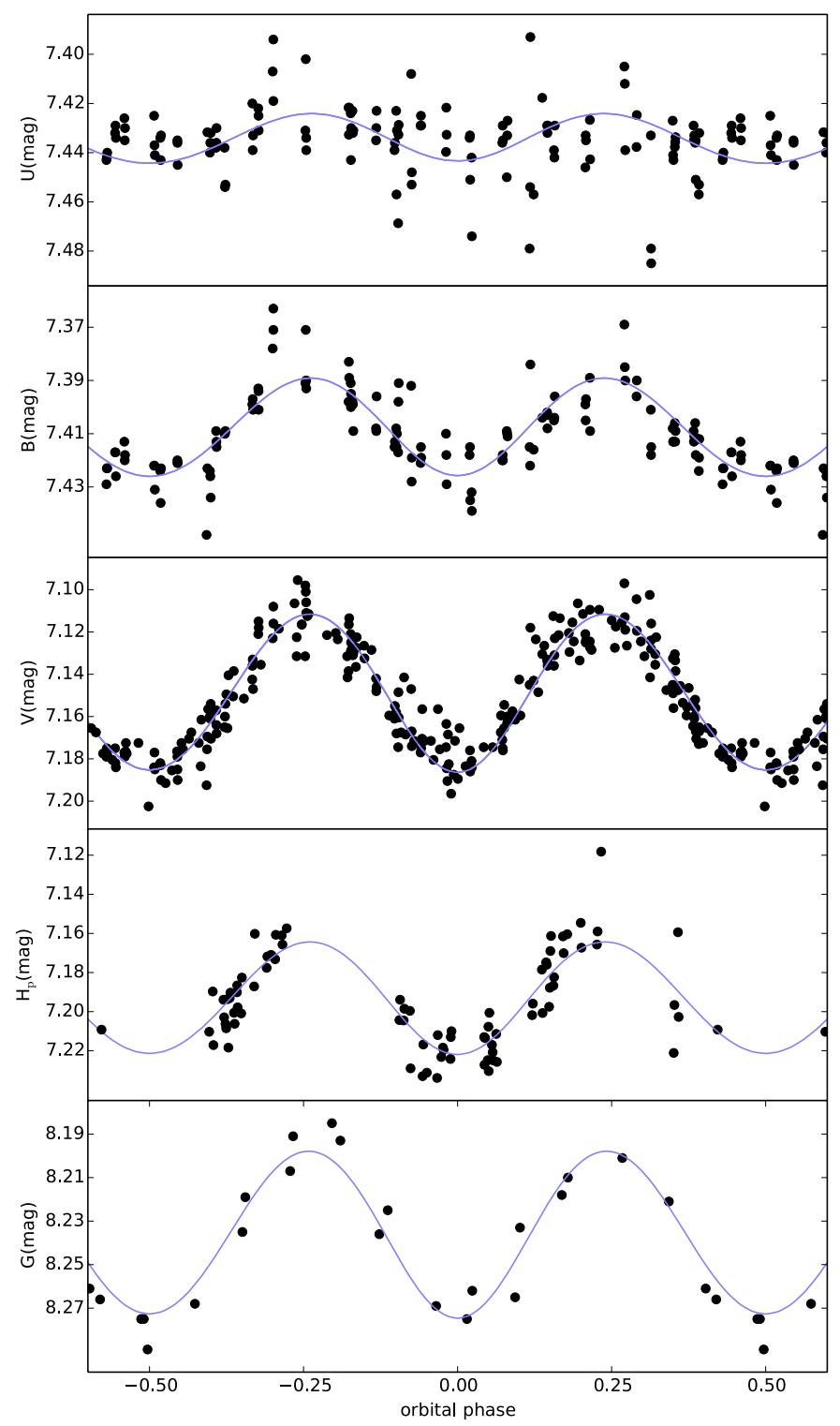

Fig. 5. Comparison of the photometric observations (black points) with the PHOEBE model light curve corresponding to the $\chi^{2}$ minimum for several photometric passbands (solid/blue line). The fits for the remaining passbands look similar. Phases from ephemeris 2 are used. Note the colour dependence of the amplitude of photometric changes on the wavelength.

PHOEBE solutions for these two subsets led to the following linear ephemerides (3) and (4)

$T_{\text {super.c. }}=\operatorname{HJD} 2450724.6695(47)+12.919018(30) \cdot E$,
$T_{\text {super.c. }}=\operatorname{HJD} 2454600.37787(30)+12^{\mathrm{d}} 919165(45) \cdot E$.

They imply a secular increase in the orbital period for $3.8 \times 10^{-8}$ days per day or $1.2 \mathrm{~s} \mathrm{yr}^{-1}$. We therefore ran another PHOEBE solution on the complete data set, this time also allowing for the convergence of the secular period change. It provided a marginal support for the reality of a secular period increase of $(3.5 \pm 1.6) \times$ $10^{-8} \mathrm{~d} \mathrm{~d}^{-1}$, the corresponding quadratic ephemeris (5) being

$$
\begin{aligned}
T_{\text {super.c. }}= & \text { HJD } 2454600.3800(22) \\
& +12.919107(26) \cdot E+2.31 \cdot 10^{-7} \cdot E^{2} .
\end{aligned}
$$

The original HIPPARCos parallax (Perryman \& ESA 1997) is $0 .^{\prime} 00670 \pm 00^{\prime} 00088$. In van Leeuwen $(2007 \mathrm{~b}, \mathrm{a})$ an 
P. Harmanec et al.: Duplicity of the Be star BR CMi

Table 7. List of some basic properties of well-observed semi-detached emission-line binaries.

\begin{tabular}{rrrrccccccc}
\hline \hline Binary & HD & Sp1+Sp2 & $\begin{array}{c}P_{\text {orb }} \\
(\mathrm{d})\end{array}$ & $\begin{array}{c}\dot{P} \\
(\mathrm{~s} / \mathrm{yr})\end{array}$ & $\begin{array}{c}\text { Cyclic } \\
\text { changes }(\mathrm{d})\end{array}$ & $\begin{array}{c}M_{1}+M_{2} \\
\left(M_{\odot}\right)\end{array}$ & $M_{2} / M_{1}$ & $r_{1} / r_{1}^{\text {Roche }}$ & $\begin{array}{c}i \\
\left({ }^{\circ}\right)\end{array}$ & $\begin{array}{c}\text { Principal } \\
\text { references }\end{array}$ \\
\hline RX Cas & - & B5:+K1III & 32.31 & +19.86 & 516.1 & $5.8+1.8$ & 0.30 & 0.06 & 80 & 1 \\
SX Cas & 232121 & B5:+K3III & 36.56 & -4.80 & $?$ & $5.1+1.5$ & 0.30 & 0.07 & 79 & 2 \\
AU Mon & 50846 & B5+G2III & 11.11 & 0.0 & 416.9 & $7.0+1.2$ & 0.17 & 0.24 & 79 & 3 \\
BR CMi & 61273 & B9.5+G8III & 12.92 & 0.0 & no & $2.31+0.14$ & 0.06 & 0.08 & 51 & 4 \\
UX Mon & 65607 & B7:+K0III & 5.904 & -0.260 & yes & $3.38+3.90$ & 1.15 & 0.36 & 81 & 5 \\
TT Hya & 97528 & B9.5+G5III & 6.953 & $0.0:$ & $?$ & $2.77+0.63$ & 0.23 & 0.17 & 83 & 6 \\
V393 Sco & 161741 & B3+A8III & 7.713 & $<0.5$ & 253.0 & $7.8+2.0$ & 0.25 & 0.25 & 80 & 7 \\
CX Dra & 174237 & B3+F5III & 6.696 & 0.0 & yes & $7.3+1.7:$ & 0.23 & 0.25 & 53 & 8 \\
$\beta$ Lyr & 174638 & B0:+B6-8II & 12.94 & +18.93 & 282.4 & $13.1+2.96$ & 0.23 & $?$ & 86 & 9 \\
V448 Cyg & 190967 & O9.5V+B1Ib & 6.520 & 0.0 & no & $24.7+13.7$ & 0.56 & 0.37 & 88 & 10 \\
V360 Lac & 216200 & B4:+F9III & 10.09 & $+5.5 ?$ & 322.2 & $7.45+1.21$ & 0.16 & 0.48 & 65 & 11 \\
\hline
\end{tabular}

Notes. In all cases, component 1 is the gainer and component 2 is the Roche-lobe-filling mass losing star. The mass ratio is always the mass of the mass-losing star over the mass of the gainer.

References. 1 Andersen et al. (1989), 2 Andersen et al. (1988), 3 Desmet et al. (2010); Atwood-Stone et al. (2012), 4 this paper, 5 Olson et al. (2009); Sudar et al. (2011), 6 Miller et al. (2007), 7 Mennickent et al. (2012a,b), 8 Koubský et al. (1998); Richards et al. (2000), 9 Harmanec et al. (1996); Bisikalo et al. (2000); Harmanec (2002); Ak et al. (2007), 10 Djurašević et al. (2009), 11 Hill et al. (1997); Linnell et al. (2006).

improved value of the HIPPARcos parallax of BR CMi, $0 .{ }^{\prime} 00579 \pm 0.00061$ is given. These values imply a distance $132-172 \mathrm{pc}$ for the original, and $156-193 \mathrm{pc}$ for the improved value. A standard dereddening of $U B V$ magnitudes of component 1 using the fractional luminosities of Table 6 gives $V_{0}=7.08,(B-V)_{0}=-0^{\mathrm{m}} 03$, and $(U-B)_{0}=-0.11$. These colours correspond to a normal B9.5 star, in agreement with the mean $T_{\text {eff }}=9685 \mathrm{~K}$ adopted as the best from the combined model fit of Table 6 for component 1 . Consulting the tabulation of normal stellar masses and radii by Harmanec (1988), we find that the mass and radius of component 1, which follow from our solution, also correspond to a normal star of spectral type B9.5-A0 V. The range of $M_{\mathrm{bol}}=1 \mathrm{~m} .01-1 \mathrm{~m} 18$ for it implies $M_{\mathrm{V}}=1.19-1^{\mathrm{m}} .36$ and a distance range of 139-151 pc. Our combined solution thus agrees with the observed distance of BR CMi. In passing, we also note that the effective temperature of component 2 , based on the comparison of model and disentangled spectra, $4655 \mathrm{~K}$, corresponds to spectral type G8 III according to a calibration by Popper (1980). The mass of component 2 is, however, much lower than the mass of a normal G8 giant, as usually found for the mass-losing stars in binaries.

\section{BR CMi among other known emission-line semi-detached binaries}

As already mentioned, the number of Be stars with known binary companions has been increasing steadily. However, many of them are single-line spectroscopic binaries and the nature of their companions is not known. There are some visual binaries among Be stars (Oudmaijer \& Parr 2010). It is not clear how strong the mutual interaction between the components of such systems can be, although there is a suspicion of strong interaction during periastron passages in some systems with highly eccentric orbits like $\delta$ Sco (cf. Meilland et al. 2013, and references therein). Another distinct subgroup of Be binaries are systems with hot and compact secondaries, the prototype of such systems being $\varphi$ Per (Gies et al. 1998; Božić et al. 1995). Only five such systems are known (see Koubský et al. 2012, 2014, and references therein). Finally, a subgroup of mass-exchanging semi-detached binaries exists in which the Be components are the mass-gaining components as predicted by the original binary hypothesis of the Be phenomenon (Kříž \& Harmanec 1975; Harmanec \& Kříž 1976).

There has also been recent progress in modelling the process of mass exchange in binaries (e.g. Siess et al. 2013; Deschamps et al. 2013; Davis et al. 2014) and obtaining reliable physical properties of mass-exchanging binaries is therefore essential for testing the predictions of new models. In Table 7, we collected a list of well-studied semi-detached emission-line binaries known to us and their published basic properties. We note that the spectral types quoted for mass gainers refer to the stellar bodies, not to the observed spectral classes affected by circumstellar matter. A zero secular change of the orbital period means that no significant change was detected in the available data.

The systems similar to those listed in Table 7 may not actually be very rare. We included only systems with reasonably safe evidence that one of the stars is filling its Roche lobe. The same might be true for the systems listed in Table 8, for which complete orbital and/or light-curve solutions and information on a secular orbital period change are still missing. Their detailed studies are very desirable. (Here, we have not included into consideration a number of short-periodic semi-detached binaries, for which transient $\mathrm{H} \alpha$ emission was reported in the astronomical literature.)

The list in Table 7 is too short to be statistically significant, but we use it to point out that there still might be a long way to a detailed comparison between observations and theory:

1. One important observable is the equatorial rotational velocity of the gainer, but it is not often available even in new studies. For instance, Mennickent et al. (2012a) considered two models in their study of V393 Sco: spin-orbit synchonisation of the gainer and gainer with a break-up velocity. Available line profiles of the gainer show that its $v \sin i$ is somewhere in between these two cases, but this piece of information was not used. Our present study of BR CMi is probably one of the few where the synchronicity parameter $F$ was changed after each iteration to correspond to the observed $v \sin i$ of the gainer.

2. It is not obvious why some of these systems exhibit cyclic (if not periodic) light and colour changes on a time scale that is an order of magnitude longer than their respective orbital periods. For three of such variables, $\beta$ Lyr, AU Mon, 
Table 8. Other possibly semi-detached emission-line binaries.

\begin{tabular}{rrcr}
\hline \hline Binary & $\begin{array}{r}\mathrm{HD} / \\
\mathrm{CoD}\end{array}$ & $\begin{array}{c}P_{\text {orb }} \\
(\mathrm{d})\end{array}$ & $\begin{array}{r}\text { Principal } \\
\text { references }\end{array}$ \\
\hline V617 Aur & 37453 & $66^{\mathrm{d}} .75$ & 1 \\
V395 Aur & 43246 & $23^{\mathrm{d}} .17$ & 2 \\
HZ CMa & 50123 & $28^{\mathrm{d}} .601$ & 3 \\
HIP 33657 & 51956 & $107^{\mathrm{d}} .4$ & 4 \\
BD-17²010 & 59771 & $\sim 90^{\mathrm{d}}$ & 5 \\
PW Pup & $-30^{\circ} 5135$ & $158^{\mathrm{d}} .0$ & 5 \\
BY Cru & 104901 & $106^{\mathrm{d}} .4$ & 6 \\
HL Lib & 127208 & $24^{\mathrm{d}} .615$ & 7 \\
V1914 Cyg & 207739 & $140^{\mathrm{d}} .782$ & 8 \\
KX And & 218393 & $38^{\mathrm{d}} 9$ & 9 \\
\hline
\end{tabular}

Notes. 1 Parsons et al. (1988); 2 Dempsey et al. (1990); 3 Sterken et al. (1994); 4 Burki \& Mayor (1983); Parsons \& Bopp (1993); 5 Eggen (1983); Bopp et al. (1991); 6 Daems et al. (1997); 7 Dempsey et al. (1990); 8 Griffin et al. (1990); Szabados (1990); 9 Štefl et al. (1990); Tarasov et al. (1998).

and V393 Sco, the presence of bipolar jets was considered. Similar jets were also considered for TT Hya, so a study of its long-term photometric behaviour would be of interest. Non-orbital light changes were also observed for UX Mon, but no clear periodicity was found. It is remarkable that UX Mon is a rare system at the early phase of the mass transfer before the mass ratio reversal. The differences in the orbital inclination also do not provide a clue. BR CMi and CX Dra are the only two non-eclipsing binaries in the considered sample, and while BR CMi seems to be secularly constant, CX Dra exhibits rather strong non-orbital long-term changes.

3. Deschamps et al. (2013) tried to classify semi-detached binaries into three classes: I. systems before the mass ratio reversal; II. systems after the reversal but with still high rate of mass transfer; and III. systems in the quiescent final stages of mass transfer. We warn that the real situation may be even more complex and note that the secular period decrease would qualify SX Cas as a candidate for class I, while its observed masses clearly show that this is a system after mass reversal. Obviously, secular period changes may also be caused by other effects than the mass transfer between the components, for instance, by the presence of other bodies in the system. We can only conclude that the results of our study provide solid evidence that BR CMi is a system at the quiescent stages of mass transfer after the mass ratio reversal. It has the lowest mass ratio of all systems of Table 7.

Acknowledgements. This study uses spectral observations obtained with the HERMES spectrograph, which is supported by the Fund for Scientific Research of Flanders (FWO), Belgium, the Research Council of K.U. Leuven, Belgium, the Fonds National de la Recherche Scientifique (F.R.S.-FNRS), Belgium, the Royal Observatory of Belgium, the Observatoire de Genève, Switzerland and the Thüringer Landessternwarte Tautenburg, Germany, spectral observations with the Elodie spectrograph attached to $1.9 \mathrm{~m}$ reflector of Haute Provence Observatory, CCD spectrograph of the Ondřejov $2 \mathrm{~m}$ reflector, and CCD spectra from the spectrograph attached to the $1.22 \mathrm{~m}$ reflector of the Dominion Astrophysical Observatory. It also uses photometric observations made at the observatory of La Palma (Mercator), Hvar, and South African Astronomical Observatory (SAAO), and by the ESA HIPPARCOS satellite. We gratefully acknowledge the use of spectrograms of BR CMi from the public archives of the Elodie spectrograph of the Haute Provence Observatory and the use of the latest publicly available versions of the programs FOTEL and KOREL written by Dr. P. Hadrava. Our sincere thanks are also due to Dr. A. Prša, who provided us with a modified version of the program PHOEBE 1.0 and frequent consultations on its usage. We thank Drs. M. Ceniga, A. Kawka, J. Kubát, P. Mayer, P. Nemeth,
M. Netolický, J. Polster, and V. Votruba, who obtained several Ondřejov spectra used in this study and to Dr. K. Uytterhoeven, who participated in photometric observations at La Palma. The comments of an anonymous referee helped to shorten the text and improve the presentation of the main results a great deal. This research was supported by the grants 205/06/0304, 205/08/H005, and P209/10/0715 of the Czech Science Foundation, by the grant 678212 of the Grant Agency of the Charles University in Prague, from the research project AV0Z10030501 of the Academy of Sciences of the Czech Republic, and from the Research Program MSM0021620860 Physical study of objects and processes in the solar system and in astrophysics of the Ministry of Education of the Czech Republic. The research of P.K. was supported from the ESA PECS grant 98058. H.B. acknowledges financial support from the Croatian Science Foundation under the project 6212 "Solar and Stellar Variability". P.N. thanks the Swiss National Science Foundation for its support in acquiring photometric data in the Geneva 7-C system. We acknowledge the use of the electronic database from the CDS, Strasbourg, and the electronic bibliography maintained by the NASA/ADS system.

\section{References}

Ak, H., Chadima, P., Harmanec, P., et al. 2007, A\&A, 463, 233

Andersen, J., Nordstrom, B., Mayor, M., \& Polidan, R. S. 1988, A\&A, 207, 37

Andersen, J., Pavlovski, K., \& Piirola, V. 1989, A\&A, 215, 272

Atwood-Stone, C., Miller, B. P., Richards, M. T., Budaj, J., \& Peters, G. J. 2012, ApJ, 760, 134

Baranne, A., Queloz, D., Mayor, M., et al. 1996, A\&AS, 119, 373

Bisikalo, D. V., Harmanec, P., Boyarchuk, A. A., Kuznetsov, O. A., \& Hadrava, P. 2000, A\&A, 353, 1009

Bopp, B. W., Dempsey, R. C., \& Parsons, S. B. 1991, PASP, 103, 444

Božić, H., Harmanec, P., Horn, J., et al. 1995, A\&A, 304, 235

Briot, D., \& Royer, F. 2009, Be Star Newsletter No. 39, 15

Burki, G., \& Mayor, M. 1983, A\&A, 124, 256

Claret, A. 1998, A\&AS, 131, 395

Claret, A. 2001, MNRAS, 327, 989

Daems, K., Waelkens, C., \& Mayor, M. 1997, A\&A, 317, 823

David, M., \& Verschueren, W. 1995, A\&AS, 111, 183

David, M., Blomme, R., Frémat, Y., et al. 2014, A\&A, 562, A97

Davis, P. J., Siess, L., \& Deschamps, R. 2014, A\&A, 570, A25

de Laverny, P., Recio-Blanco, A., Worley, C. C., \& Plez, B. 2012, A\&A, 544, A126

Dempsey, R. C., Bopp, B. W., Parsons, S. B., \& Fekel, F. C. 1990, PASP, 102, 312

Deschamps, R., Siess, L., Davis, P. J., \& Jorissen, A. 2013, A\&A, 557, A40

Desmet, M., Frémat, Y., Baudin, F., et al. 2010, MNRAS, 401, 418

Djurašević, G., Vince, I., Khruzina, T. S., \& Rovithis-Livaniou, E. 2009, MNRAS, 396, 1553

Dubath, P., Rimoldini, L., Süveges, M., et al. 2011, MNRAS, 414, 2602

Eaton, J. A. 2008, ApJ, 681, 562

Eggen, O. J. 1983, AJ, 88, 1676

Gies, D. R., Bagnuolo, Jr., W. G., Ferrara, E. C., et al. 1998, ApJ, 493, 440

Griffin, R. F., Parsons, S. B., Dempsey, R., \& Bopp, B. W. 1990, PASP, 102, 535

Hadrava, P. 1990, Contr. Astron. Obs. Skalnaté Pleso, 20, 23

Hadrava, P. 1995, A\&AS, 114, 393

Hadrava, P. 2004a, Publ. Astron. Inst. Acad. Sci. Czech Rep., 92, 1

Hadrava, P. 2004b, Publ. Astron. Inst. Acad. Sci. Czech Rep., 92, 15

Harmanec, P. 1988, Bull. Astron. Inst. Czechosl., 39, 329

Harmanec, P. 1998, A\&A, 335, 173

Harmanec, P. 2002, Astron. Nachr., 323, 87

Harmanec, P. 2003, in Publ. Canakkale Onsekiz Mart Univ.: New Directions for Close Binary Studies: The Royal Road to the Stars, 3, 221

Harmanec, P., \& Horn, J. 1998, J. Astron. Data, 4, 5

Harmanec, P., \& Kříž, S. 1976, in Be and Shell Stars, ed. A. Slettebak, IAU Symp., 70, 385

Harmanec, P., \& Scholz, G. 1993, A\&A, 279, 131

Harmanec, P., Horn, J., \& Juza, K. 1994, A\&AS, 104, 121

Harmanec, P., Morand, F., Bonneau, D., et al. 1996, A\&A, 312, 879

Hill, G., Harmanec, P., Pavlovski, K., et al. 1997, A\&A, 324, 965

Horn, J., Kubát, J., Harmanec, P., et al. 1996, A\&A, 309, 521

Kazarovets, E. V., Samus, N. N., Durlevich, O. V., et al. 1999, IBVS, 4659, 1

Koubský, P., Harmanec, P., Božić, H., et al. 1998, Hvar Observatory Bulletin, 22, 17

Koubský, P., Kotková, L., Votruba, V., Šlechta, M., \& Dvořáková, Š. 2012, A\&A, 545, A121

Koubský, P., Kotková, L., Kraus, M., et al. 2014, A\&A, 567, A57

Kř́̌ž, S., \& Harmanec, P. 1975, BAICz, 26, 65 
Linnell, A. P., Harmanec, P., Koubský, P. et al. 2006, A\&A, 455, 1037

Lucy, L. B., \& Sweeney, M. A. 1971, AJ, 76, 544

Meilland, A., Stee, P., Spang, A., et al. 2013, A\&A, 550, L5

Mennickent, R. E., Djurašević, G., Kołaczkowski, Z., \& Michalska, G. 2012a, MNRAS, 421, 862

Mennickent, R. E., Kołaczkowski, Z., Djurašević, G., et al. 2012b, MNRAS, 427, 607

Miller, B., Budaj, J., Richards, M., Koubský, P., \& Peters, G. J. 2007, ApJ, 656, 1075

Moore, C. E. 1945, Contributions from the Princeton University Observatory, 20, D23

Nasseri, A., Chini, R., Harmanec, P., et al. 2014, A\&A, 568, A94

Olson, E. C., Henry, G. W., \& Etzel, P. B. 2009, AJ, 138, 1435

Oudmaijer, R. D., \& Parr, A. M. 2010, MNRAS, 405, 2439

Palacios, A., Gebran, M., Josselin, E., et al. 2010, A\&A, 516, A13

Parsons, S. B., \& Bopp, B. W. 1993, in American Astronomical Society Meeting Abstracts, BAAS, 25, 1376

Parsons, S. B., Dempsey, R. C., \& Bopp, B. W. 1988, in ESA SP, 281, 225

Paunzen, E., Duffee, B., Heiter, U., Kuschnig, R., \& Weiss, W. W. 2001, A\&A, 373, 625

Perryman, M. A. C., \& ESA 1997, The HiPPARCos and Tycho catalogues, Astrometric and photometric star catalogues derived from the ESA Hipparcos Space Astrometry Mission (Noordwijk: ESA Publications Division) ESA SP Series 1200

Peters, G. J., Pewett, T. D., Gies, D. R., Touhami, Y. N., \& Grundstrom, E. D. 2013, ApJ, 765, 2

Pojmanski, G. 2002, Acta Astron., 52, 397

Popper, D. M. 1980, ARA\&A, 18, 115
Press, W. H., Teukolsky, S. A., Vetterling, W. T., \& Flannery, B. P. 1993, Numerical Recipes in FORTRAN; The Art of Scientific Computing, 2nd edn. (New York: Cambridge University Press)

Prša, A., \& Zwitter, T. 2005, ApJ, 628, 426

Prša, A., \& Zwitter, T. 2006, Ap\&SS, 36

Raskin, G., Burki, G., Burnet, M., et al. 2004, in Ground-based Instrumentation for Astronomy, eds. A. F. M. Moorwood, \& M. Iye, SPIE Conf. Ser., 5492, 830

Raskin, G., van Winckel, H., Hensberge, H., et al. 2011, A\&A, 526, A69

Renson, P., \& Manfroid, J. 2009, A\&A, 498, 961

Richards, M. T., Koubský, P., Šimon, V., et al. 2000, ApJ, 531, 1003

Royer, F., Briot, D., North, P., Burki, G., \& Carrier, F. 2007, in IAU Symp. 240, eds. W. I. Hartkopf, E. F. Guinan, \& P. Harmanec, 211

Ruždjak, D., Božić, H., Harmanec, P., et al. 2009, A\&A, 506, 1319

Siess, L., Izzard, R. G., Davis, P. J., \& Deschamps, R. 2013, A\&A, 550, A100

Škoda, P. 1996, in Astronomical Data Analysis Software and Systems V, ASP Conf. Ser., 101, 187

Štefl, S., Harmanec, P., Horn, J., et al. 1990, BAICz, 41, 29

Sterken, C., Vogt, N., \& Mennickent, R. 1994, A\&A, 291, 473

Sterne, T. E. 1941, PNAS, 27, 168

Stetson, P. B. 1991, AJ, 102, 589

Sudar, D., Harmanec, P., Lehmann, H., et al. 2011, A\&A, 528, A146

Szabados, L. 1990, A\&A, 232, 381

Tarasov, A. E., Berdyugina, S. V., \& Berdyugin, A. V. 1998, Astron. Lett., 24, 316

van Leeuwen, F. 2007a, in Astrophys. Space Sci. Lib. 350 (Berlin: Springer)

van Leeuwen, F. 2007b, A\&A, 474, 653

Zucker, S., \& Mazeh, T. 1994, ApJ, 420, 806 


\section{Appendix A: Details of the spectral data reduction and measurements}

The initial reduction of all Ondřejov and DAO spectra (bias subtraction, flat-fielding, creation of 1-D spectra, and wavelength calibration) was carried out in IRAF. For Elodie and Hermes spectra, dedicated reduction pipelines were used, combined with some IDL routines in the case of Elodie. See Baranne et al. (1996) and Raskin et al. (2011) for detailed descriptions of the Elodie and Hermes spectrographs. Rectification, removal of residual cosmics and flaws and RV measurements of all spectra were carried out with the program SPEF0 (Horn et al. 1996; Škoda 1996), namely the latest version 2.63 developed by Mr. J. Krpata. SPEFO displays direct and flipped traces of the line profiles superimposed on the computer screen that the user can slide to achieve a precise overlapping of the parts of the profile of whose RV one wants to measure. Using a selection of stronger unblended lines of the cool component 2 (see Table A.1) covering the red spectral region (available for all spectra), we measured RVs of all of them to obtain a mean RV for all spectra. We also measured a selection of good telluric lines and used them for an additional fine correction of the RV zero point of each spectrogram (Horn et al. 1996). Moreover, we measured the RVs of the steep wings of the $\mathrm{H} \alpha$ emission line and of the two absorption cores of $\mathrm{H} \alpha$. We point out that although some broad and shallow lines of component 1 are seen in the spectra, their direct RV measurement is impossible because of numerous blends with the lines of component 2 .

Table A.1. Spectral lines of component 2 and their air wavelengths used for the RV measurements in SPEFO.

\begin{tabular}{clcl}
\hline $\begin{array}{c}\text { Wavelength } \\
(\AA)\end{array}$ & Element & $\begin{array}{c}\text { Wavelength } \\
(\AA)\end{array}$ & Element \\
\hline 6322.694 & Fe I 207 & 6439.083 & Ca I 18 \\
6327.604 & Ni I 49 & 6643.638 & Ni I 43 \\
6355.035 & Fe I 342 & 6677.997 & Fe I 268 \\
6358.687 & Fe I 13 & 6705.105 & Fe I 1 197 \\
6393.612 & Fe I 168 & 6717.687 & Ca I 32 \\
6411.658 & Fe I 816 & 6726.673 & Fe I 1 197 \\
6430.856 & Fe I 62 & & \\
\hline
\end{tabular}

Notes. The numbering of the multiplets of individual ions corresponds to the one introduced by Moore (1945). 
P. Harmanec et al.: Duplicity of the Be star BR CMi

Table A.2. Individual SPEFO RVs of component 2.

\begin{tabular}{|c|c|c|c|c|c|c|c|c|}
\hline $\begin{array}{c}\text { HJD } \\
-2400000\end{array}$ & $\begin{array}{r}\mathrm{RV} \\
\left(\mathrm{km} \mathrm{s}^{-1}\right)\end{array}$ & Spg. & $\begin{array}{c}\text { HJD } \\
-2400000\end{array}$ & $\begin{array}{r}\mathrm{RV} \\
\left(\mathrm{km} \mathrm{s}^{-1}\right)\end{array}$ & Spg. & $\begin{array}{c}\text { HJD } \\
-2400000\end{array}$ & $\begin{array}{r}\mathrm{RV} \\
\left(\mathrm{km} \mathrm{s}^{-1}\right)\end{array}$ & Spg. \\
\hline 50071.5415 & 17.71 & E & 54115.5309 & 2.80 & $\mathrm{O}$ & 55592.4540 & -98.74 & $\mathrm{O}$ \\
\hline 50072.5331 & -27.10 & $\mathrm{E}$ & 54116.4556 & -38.40 & $\mathrm{O}$ & 55593.3881 & -82.17 & $\mathrm{O}$ \\
\hline 50527.3026 & -101.63 & $\mathrm{E}$ & 54172.3849 & -78.18 & $\mathrm{O}$ & 55601.5399 & -10.33 & $\mathrm{O}$ \\
\hline 50528.2947 & -98.11 & E & 54173.4495 & -38.80 & $\mathrm{O}$ & 55602.5009 & -52.10 & $\mathrm{O}$ \\
\hline 50529.2966 & -76.42 & $\mathrm{E}$ & 54174.4208 & 2.48 & $\mathrm{O}$ & 55613.5249 & 29.96 & $\mathrm{O}$ \\
\hline 50530.3328 & -37.71 & E & 54176.4755 & 67.45 & $\mathrm{O}$ & 55614.4452 & -7.48 & $\mathrm{O}$ \\
\hline 50842.5007 & 46.40 & E & 54185.3680 & -74.68 & $\mathrm{O}$ & 55621.4578 & 6.53 & $\mathrm{O}$ \\
\hline 50843.6757 & 72.95 & E & 54185.3914 & -74.08 & $\mathrm{O}$ & 55623.4517 & 67.26 & $\mathrm{O}$ \\
\hline 51565.4951 & 30.44 & E & 54185.4381 & -74.44 & $\mathrm{O}$ & 55628.4816 & -59.02 & $\mathrm{O}$ \\
\hline 51567.4662 & 76.03 & E & 54186.3577 & -39.80 & $\mathrm{O}$ & 55644.4341 & -96.59 & $\mathrm{O}$ \\
\hline 52215.6544 & 34.01 & E & 54193.4186 & -13.47 & $\mathrm{O}$ & 55645.4161 & -70.53 & $\mathrm{O}$ \\
\hline 52216.6342 & -5.82 & E & 54197.3561 & -98.74 & $\mathrm{O}$ & 55649.4090 & 70.69 & $\mathrm{O}$ \\
\hline 52217.6902 & -51.60 & E & 54205.3780 & 25.44 & $\mathrm{O}$ & 54733.0084 & 74.78 & D \\
\hline 52220.6997 & -97.73 & $\mathrm{E}$ & 54206.4006 & -15.76 & $\mathrm{O}$ & 54939.6777 & 74.31 & $\mathrm{D}$ \\
\hline 53007.5331 & -100.19 & E & 54211.3746 & -69.48 & $\mathrm{O}$ & 54939.7269 & 74.40 & D \\
\hline 53008.5693 & -101.34 & E & 54222.3531 & -104.84 & $\mathrm{O}$ & 54942.6759 & -11.64 & D \\
\hline 53009.5262 & -82.21 & E & 54384.5600 & 71.98 & $\mathrm{O}$ & 55469.0305 & 74.34 & $\mathrm{D}$ \\
\hline 53010.5413 & -47.46 & E & 54388.6316 & -71.52 & $\mathrm{O}$ & 55471.0483 & 41.72 & D \\
\hline 53011.6043 & -3.39 & $\mathrm{E}$ & 54508.4493 & -74.42 & $\mathrm{O}$ & 55570.0131 & 15.43 & $\mathrm{D}$ \\
\hline 53014.4313 & 73.96 & $\mathrm{E}$ & 54535.4177 & -30.08 & $\mathrm{O}$ & 55501.0092 & -101.26 & $\mathrm{D}$ \\
\hline 53413.5151 & 50.38 & $\mathrm{E}$ & 54569.3620 & -67.56 & $\mathrm{O}$ & 55601.9629 & -29.49 & $\mathrm{D}$ \\
\hline 53414.4822 & 71.49 & $\mathrm{E}$ & 54840.5091 & -61.06 & $\mathrm{O}$ & 55602.9492 & -68.74 & D \\
\hline 53416.3923 & 57.77 & E & 54932.3291 & -98.41 & $\mathrm{O}$ & 55858.0824 & 58.42 & D \\
\hline 53418.4050 & -19.47 & E & 54934.3986 & -83.54 & $\mathrm{O}$ & 55953.9157 & -101.92 & $\mathrm{D}$ \\
\hline 53419.4041 & -61.42 & E & 54937.3221 & 27.71 & $\mathrm{O}$ & 55993.7744 & -84.07 & $\mathrm{D}$ \\
\hline 53420.3829 & -90.60 & E & 55274.4052 & 64.25 & $\mathrm{O}$ & 55994.7681 & -50.46 & D \\
\hline 53421.3983 & -102.84 & $\mathrm{E}$ & 55278.2936 & 0.91 & $\mathrm{O}$ & 56031.7031 & -99.73 & $\mathrm{D}$ \\
\hline 53422.4619 & -93.35 & $\mathrm{E}$ & 55280.3356 & -81.24 & $\mathrm{O}$ & 56296.0462 & 74.91 & $\mathrm{D}$ \\
\hline 53424.4110 & -28.41 & $\mathrm{E}$ & 55281.4343 & -102.20 & $\mathrm{O}$ & 56381.7600 & -71.06 & D \\
\hline 53425.4095 & 13.94 & $\mathrm{E}$ & 55293.3180 & -82.46 & $\mathrm{O}$ & 56382.7638 & -32.61 & $\mathrm{D}$ \\
\hline 53426.4032 & 50.64 & E & 55294.3667 & -100.89 & $\mathrm{O}$ & 56383.7508 & 9.30 & $\mathrm{D}$ \\
\hline 53428.4087 & 72.95 & E & 55295.3084 & -101.04 & $\mathrm{O}$ & 55589.5585 & -51.62 & $\mathrm{H}$ \\
\hline 53429.3832 & 55.55 & $\mathrm{E}$ & 55296.3068 & -79.51 & $\mathrm{O}$ & 55592.5374 & -98.92 & $\mathrm{H}$ \\
\hline 53430.3826 & 21.99 & $\mathrm{E}$ & 55315.3200 & 61.49 & $\mathrm{O}$ & 55595.4975 & 0.26 & $\mathrm{H}$ \\
\hline 54000.6311 & -56.47 & $\mathrm{O}$ & 55480.6300 & 54.66 & $\mathrm{O}$ & 55904.7369 & -34.63 & $\mathrm{H}$ \\
\hline 54017.6633 & -68.43 & $\mathrm{O}$ & 55491.5525 & -24.09 & $\mathrm{O}$ & 55906.6854 & 43.68 & $\mathrm{H}$ \\
\hline 54018.6197 & -31.83 & $\mathrm{O}$ & 55578.4610 & -100.52 & $\mathrm{O}$ & 55908.6513 & 74.17 & $\mathrm{H}$ \\
\hline 54019.5490 & 7.60 & $\mathrm{O}$ & 55579.5032 & -100.38 & $\mathrm{O}$ & 55910.6683 & 28.73 & $\mathrm{H}$ \\
\hline 54025.5356 & -17.63 & $\mathrm{O}$ & 55590.4031 & -81.03 & $\mathrm{O}$ & 55912.7053 & -58.62 & $\mathrm{H}$ \\
\hline
\end{tabular}

Notes. Letters E, O, D, and H in column "Spg." denote the Elodie, Ondřejov, DAO, and Hermes spectrographs. 
Table A.3. Individual asTODCOR RVs of both components.

\begin{tabular}{crrrrrrr}
\hline \hline $\begin{array}{c}\mathrm{HJD} \\
-2400000\end{array}$ & $\begin{array}{r}\mathrm{RV}_{1} \\
\left(\mathrm{~km} \mathrm{~s}^{-1}\right)\end{array}$ & $\begin{array}{r}\mathrm{RV}_{2} \\
\left(\mathrm{~km} \mathrm{~s}^{-1}\right)\end{array}$ & weight & $\begin{array}{c}\mathrm{HJD} \\
-2400000\end{array}$ & $\begin{array}{r}\mathrm{RV}_{1} \\
\left(\mathrm{~km} \mathrm{~s}^{-1}\right)\end{array}$ & $\begin{array}{r}\mathrm{RV}_{2} \\
\left(\mathrm{~km} \mathrm{~s}^{-1}\right)\end{array}$ & Weight \\
\hline 50071.5415 & -1.160 & 31.356 & 0.538 & 53416.3923 & -4.494 & 71.609 & 0.424 \\
50072.5331 & 1.731 & -12.346 & 0.842 & 53418.4050 & 0.610 & -4.722 & 0.968 \\
50527.3026 & 4.675 & -87.466 & 0.840 & 53419.4041 & 3.331 & -46.735 & 0.765 \\
50528.2947 & 5.177 & -83.880 & 0.655 & 53420.3829 & 4.181 & -76.069 & 0.988 \\
50529.2966 & 4.325 & -60.928 & 0.706 & 53421.3983 & 4.300 & -88.491 & 0.915 \\
50530.3328 & 1.951 & -22.894 & 1.333 & 53422.4619 & 4.515 & -78.451 & 0.426 \\
50842.5007 & -3.226 & 60.359 & 1.302 & 53424.4110 & 0.773 & -13.666 & 0.688 \\
51565.4951 & -2.191 & 43.976 & 0.503 & 53425.4095 & -2.012 & 28.347 & 0.680 \\
51567.4662 & -4.117 & 88.335 & 0.440 & 53426.4032 & -2.985 & 63.936 & 0.511 \\
52215.6544 & -3.967 & 48.493 & 0.858 & 53428.4087 & -5.194 & 86.918 & 0.872 \\
52216.6342 & -1.243 & 8.344 & 1.335 & 53429.3832 & -4.271 & 69.230 & 0.318 \\
52217.6902 & 1.581 & -37.213 & 0.781 & 53430.3826 & -2.161 & 36.197 & 0.850 \\
52220.6997 & 5.196 & -83.691 & 0.497 & 55589.5585 & 3.450 & -37.172 & 1.578 \\
53007.5331 & 4.374 & -85.632 & 1.310 & 55592.5374 & 6.050 & -84.350 & 0.840 \\
53008.5693 & 4.292 & -86.321 & 0.761 & 55595.4975 & -0.645 & 14.860 & 1.345 \\
53009.5262 & 4.938 & -67.760 & 1.824 & 55904.7369 & 1.693 & -19.898 & 1.590 \\
53010.5413 & 2.655 & -32.753 & 1.185 & 55906.6854 & -3.181 & 57.918 & 1.406 \\
53011.6043 & -1.166 & 11.645 & 1.440 & 55908.6513 & -4.222 & 88.134 & 1.124 \\
53014.4313 & -5.432 & 88.287 & 1.501 & 55910.6683 & -3.695 & 42.479 & 1.566 \\
53413.5151 & -3.674 & 65.533 & 0.359 & 55912.7053 & 4.324 & -43.761 & 1.270 \\
53414.4822 & -5.642 & 84.895 & 2.866 & & & & \\
\hline
\end{tabular}

Notes. The weights are proportional to the square of the $\mathrm{S} / \mathrm{N}$ of individual spectra.

\section{Appendix B: Details on the photometric data reductions}

Since we used photometry from various sources and photometric systems, both all-sky and differential, relative to several different comparison stars, we attempted to arrive at some homogenisation and standardisation. Special effort was made to derive improved all-sky values for the comparison stars used, employing carefully standardised $U B V$ observations secured at Hvar over several decades of systematic observations. The adopted values are collected in Table B.1, together with the number of all-sky observations and the rms errors of one observation. They were added to the respective magnitude differences to obtain directly comparable standard $U B V$ magnitudes for all stations. To illustrate the accuracy, with which various data sets were transformed to the standard system, we give (in Table B.2) mean differential $U B V$ values for the check stars used. They were derived relatively to the Hvar values for the comparison stars HD 58187 and HD 61341.

Below, we provide some details of the individual data sets and their reductions.

- Station 01 - Hvar: these differential observations have been secured by $\mathrm{HB}$ and PZ relative to HD 58187 (the check star HD 59059 being observed as frequently as the variable) and carefully transformed to the standard $U B V$ system via non-linear transformation formulæ using the HEC22 reduction program - see Harmanec et al. (1994) and Harmanec \& Horn (1998) for the observational strategy and data reduction ${ }^{3}$. All observations were reduced with the latest HEC22 rel. 17 program, which allows the time variation of linear extinction coefficients to be modelled in the course of observing nights.

- Station 11 - South African Astronomical Observatory (SAAO): these differential $U B V$ observations were obtained by $\mathrm{PZ}$ with the $0.50 \mathrm{~m}$ reflector relative to HD 61341 (SAO 115750 being used as the check star) and also transformed to the standard Johnson system with the help of HEC22.

- Station 37 - La Palma: these all-sky seven-colour (7-C) observations were secured in the Geneva photometric system using the two-channel aperture photometer P7-2000 (Raskin et al. 2004) mounted on the $1.20 \mathrm{~m}$ Belgian Mercator reflector at the Observatorio de los Muchachos at La Palma.

- Station 61 - HIPPARCos: these all-sky observations were reduced to the standard $V$ magnitude via the transformation formulæ derived by Harmanec (1998) to verify that no secular light changes in the system were observed. However, for the light-curve solution in PHOEBE, we consider the HIPPARCOS transmission curve for the $H_{\mathrm{p}}$ magnitude.

- Station 93 - ASAS3 V photometry: we extracted these allsky observations from the ASAS3 public archive (Pojmanski 2002), using the data for diaphragm 1, having on average the lowest rms errors. We omitted all observations of grade D and observations having rms errors larger than 0 . 04 . We also omitted a strongly deviating observation at HJD 2452662.6863.

\footnotetext{
3 The whole program suite with a detailed manual, examples of data, auxiliary data files, and results is available at http://astro.troja. mff.cuni.cz/ftp/hec/PHOT
} 
P. Harmanec et al.: Duplicity of the Be star BR CMi

Table B.1. Accurate Hvar and SAAO all-sky mean $U B V$ values for all comparison stars used.

\begin{tabular}{rrrrcccrr}
\hline \hline Station & Star & HD & $\begin{array}{r}\text { No. of } \\
\text { obs. }\end{array}$ & $\begin{array}{c}V \\
\text { (mag.) }\end{array}$ & $\begin{array}{c}B \\
\text { (mag.) }\end{array}$ & $\begin{array}{c}U \\
\text { (mag.) }\end{array}$ & $\begin{array}{r}(B-V) \\
\text { (mag.) }\end{array}$ & $\begin{array}{r}(U-B) \\
\text { (mag.) }\end{array}$ \\
\hline 01 & 1 CMi & 58187 & 466 & $5.393 \pm 0.010$ & $5.493 \pm 0.011$ & $5.622 \pm 0.012$ & 0.100 & 0.129 \\
01 & HR 2858 & 59059 & 364 & $6.254 \pm 0.010$ & $6.206 \pm 0.011$ & $6.103 \pm 0.013$ & -0.048 & -0.103 \\
01 & SAO 115753 & 61341 & 39 & $7.991 \pm 0.010$ & $8.182 \pm 0.009$ & $8.281 \pm 0.024$ & 0.191 & 0.099 \\
\hline 11 & SAO 115753 & 61341 & 3 & $7.986 \pm 0.010$ & $8.178 \pm 0.013$ & $8.288 \pm 0.015$ & 0.192 & 0.110 \\
11 & SAO 115750 & - & 24 & $9.743 \pm 0.011$ & $9.883 \pm 0.013$ & $10.012 \pm 0.018$ & 0.139 & 0.129 \\
\hline
\end{tabular}

Notes. These were added to the magnitude differences var.-comp. and check-comp. for data from all stations.

Table B.2. Mean $U B V$ values for the check stars used at individual observing stations derived differentially relative to their respective comparison stars.

\begin{tabular}{rrrrcccrr}
\hline \hline Station & Star & HD & $\begin{array}{r}\text { No. of } \\
\text { obs. }\end{array}$ & $\begin{array}{c}V \\
\text { (mag.) }\end{array}$ & $\begin{array}{c}B \\
\text { (mag.) }\end{array}$ & $\begin{array}{c}U \\
\text { (mag.) }\end{array}$ & $\begin{array}{r}(B-V) \\
\text { (mag.) }\end{array}$ & $\begin{array}{r}(U-B) \\
\text { (mag.) }\end{array}$ \\
\hline 01 & HR 2858 & 59059 & 542 & $6.254 \pm 0.010$ & $6.206 \pm 0.009$ & $6.102 \pm 0.013$ & -0.048 & -0.104 \\
01 & SAO 115753 & 61341 & 53 & $7.987 \pm 0.013$ & $8.182 \pm 0.011$ & $8.278 \pm 0.023$ & 0.195 & 0.096 \\
\hline 11 & SAO 115750 & - & 28 & $9.747 \pm 0.009$ & $9.886 \pm 0.011$ & $10.004 \pm 0.013$ & 0.140 & 0.118 \\
\hline
\end{tabular}

Notes. They illustrate how closely the data could be transformed to a comparable standard system.

Table B.3. Individual Geneva 7-C observations.

\begin{tabular}{cccccccc}
\hline \hline $\begin{array}{c}\text { HJD } \\
-2400000\end{array}$ & $\begin{array}{c}U \\
(\mathrm{mag})\end{array}$ & $\begin{array}{c}B \\
(\mathrm{mag})\end{array}$ & $\begin{array}{c}V \\
(\mathrm{mag})\end{array}$ & $\begin{array}{c}B 1 \\
(\mathrm{mag})\end{array}$ & $\begin{array}{c}B 2 \\
(\mathrm{mag})\end{array}$ & $\begin{array}{c}V 1 \\
(\mathrm{mag})\end{array}$ & $\begin{array}{c}G \\
(\mathrm{mag})\end{array}$ \\
\hline 52307.44605 & 7.881 & 6.495 & 7.173 & 7.417 & 7.940 & 7.894 & 8.275 \\
52307.55703 & 7.876 & 6.486 & 7.159 & 7.409 & 7.931 & 7.876 & 8.262 \\
52308.45517 & 7.900 & 6.497 & 7.161 & 7.426 & 7.938 & 7.876 & 8.265 \\
52308.55777 & 7.881 & 6.472 & 7.130 & 7.404 & 7.904 & 7.846 & 8.233 \\
52309.43489 & 7.892 & 6.480 & 7.126 & 7.414 & 7.915 & 7.847 & 8.218 \\
52309.56210 & 7.886 & 6.474 & 7.112 & 7.403 & 7.905 & 7.832 & 8.210 \\
52312.45134 & 7.894 & 6.492 & 7.154 & 7.418 & 7.935 & 7.878 & 8.261 \\
52313.53300 & 7.907 & 6.503 & 7.171 & 7.426 & 7.945 & 7.890 & 8.275 \\
52317.53358 & 7.867 & 6.455 & 7.092 & 7.387 & 7.886 & 7.811 & 8.185 \\
52318.52657 & 7.887 & 6.484 & 7.135 & 7.412 & 7.918 & 7.859 & 8.236 \\
52339.42680 & 7.900 & 6.502 & 7.177 & 7.426 & 7.939 & 7.893 & 8.275 \\
52350.43584 & 7.881 & 6.475 & 7.130 & 7.400 & 7.913 & 7.851 & 8.221 \\
52351.42891 & 7.901 & 6.500 & 7.163 & 7.425 & 7.934 & 7.888 & 8.266 \\
52352.42504 & 7.899 & 6.503 & 7.178 & 7.431 & 7.947 & 7.898 & 8.289 \\
52353.41762 & 7.893 & 6.497 & 7.164 & 7.423 & 7.938 & 7.885 & 8.268 \\
52354.41404 & 7.888 & 6.481 & 7.133 & 7.418 & 7.918 & 7.856 & 8.235 \\
52355.41486 & 7.885 & 6.474 & 7.108 & 7.409 & 7.905 & 7.832 & 8.207 \\
52362.37901 & 7.881 & 6.470 & 7.107 & 7.400 & 7.905 & 7.828 & 8.201 \\
52367.39349 & 7.861 & 6.458 & 7.119 & 7.386 & 7.901 & 7.841 & 8.219 \\
52368.39548 & 7.880 & 6.461 & 7.095 & 7.396 & 7.895 & 7.818 & 8.191 \\
52369.38697 & 7.873 & 6.461 & 7.095 & 7.392 & 7.891 & 7.817 & 8.193 \\
52370.38003 & 7.877 & 6.474 & 7.127 & 7.399 & 7.908 & 7.848 & 8.225 \\
52371.39401 & 7.897 & 6.500 & 7.165 & 7.421 & 7.939 & 7.885 & 8.269 \\
52384.38727 & 7.890 & 6.486 & 7.152 & 7.411 & 7.925 & 7.869 & 8.264 \\
\hline & & & & & & & \\
\hline & & & & & & \\
523 &
\end{tabular}


A\&A 573, A107 (2015)

Table B.4. Original HIPPARCos $H_{\mathrm{p}}$ observations and their conversion to $V$ band observations.

\begin{tabular}{|c|c|c|c|c|c|c|c|}
\hline $\begin{array}{c}\text { HJD } \\
-2400000 \\
\end{array}$ & $\begin{array}{c}H_{\mathrm{p}} \\
(\mathrm{mag})\end{array}$ & $\begin{array}{c}\mathrm{rms} \\
(\mathrm{mag})\end{array}$ & $\begin{array}{c}V \\
\text { (mag) }\end{array}$ & HJD & $\begin{array}{c}H_{\mathrm{p}} \\
(\mathrm{mag})\end{array}$ & $\begin{array}{c}\mathrm{rms} \\
(\mathrm{mag})\end{array}$ & $\begin{array}{c}V \\
(\mathrm{mag})\end{array}$ \\
\hline 47966.1374 & 7.2184 & 0.007 & 7.157 & 48393.5983 & 7.2257 & 0.008 & 7.164 \\
\hline 47966.1517 & 7.2195 & 0.007 & 7.158 & 48404.6932 & 7.1996 & 0.012 & 7.138 \\
\hline 47987.3742 & 7.1939 & 0.009 & 7.133 & 48404.7075 & 7.2290 & 0.008 & 7.168 \\
\hline 47987.3885 & 7.2029 & 0.010 & 7.142 & 48404.9599 & 7.2330 & 0.014 & 7.172 \\
\hline 47987.4630 & 7.1936 & 0.007 & 7.132 & 48404.9742 & 7.2168 & 0.008 & 7.156 \\
\hline 47987.4774 & 7.2184 & 0.008 & 7.157 & 48405.0487 & 7.2312 & 0.014 & 7.170 \\
\hline 48013.4952 & 7.1901 & 0.007 & 7.129 & 48569.9541 & 7.1610 & 0.006 & 7.100 \\
\hline 48013.5095 & 7.1978 & 0.007 & 7.137 & 48569.9684 & 7.1657 & 0.013 & 7.104 \\
\hline 48013.5840 & 7.2009 & 0.009 & 7.140 & 48570.0573 & 7.1574 & 0.011 & 7.096 \\
\hline 48013.5984 & 7.1826 & 0.009 & 7.121 & 48586.1305 & 7.2339 & 0.010 & 7.173 \\
\hline 48147.8756 & 7.2272 & 0.008 & 7.166 & 48586.1449 & 7.2120 & 0.008 & 7.151 \\
\hline 48147.8900 & 7.2135 & 0.006 & 7.152 & 48586.2194 & 7.2232 & 0.009 & 7.162 \\
\hline 48147.9646 & 7.2304 & 0.008 & 7.169 & 48586.4115 & 7.2242 & 0.007 & 7.163 \\
\hline 48147.9789 & 7.2006 & 0.007 & 7.139 & 48705.7530 & 7.1657 & 0.008 & 7.104 \\
\hline 48148.0534 & 7.2249 & 0.008 & 7.164 & 48705.7673 & 7.1590 & 0.008 & 7.098 \\
\hline 48164.7628 & 7.2211 & 0.009 & 7.160 & 48705.8418 & 7.1182 & 0.013 & 7.057 \\
\hline 48164.7771 & 7.1966 & 0.007 & 7.135 & 48731.2523 & 7.1546 & 0.006 & 7.093 \\
\hline 48164.8517 & 7.1594 & 0.016 & 7.098 & 48731.2666 & 7.1673 & 0.006 & 7.106 \\
\hline 48164.8660 & 7.2027 & 0.010 & 7.141 & 48753.2881 & 7.2043 & 0.007 & 7.143 \\
\hline 48194.3522 & 7.1997 & 0.007 & 7.138 & 48753.3025 & 7.1939 & 0.006 & 7.133 \\
\hline 48194.3665 & 7.1866 & 0.007 & 7.125 & 48753.3770 & 7.2045 & 0.009 & 7.143 \\
\hline 48316.8239 & 7.2018 & 0.013 & 7.141 & 48753.3913 & 7.1985 & 0.008 & 7.137 \\
\hline 48316.8382 & 7.1959 & 0.010 & 7.135 & 48935.2322 & 7.2130 & 0.008 & 7.152 \\
\hline 48317.1794 & 7.1975 & 0.010 & 7.136 & 48935.2465 & 7.2100 & 0.008 & 7.149 \\
\hline 48317.1937 & 7.1878 & 0.011 & 7.127 & 49047.3806 & 7.1871 & 0.012 & 7.126 \\
\hline 48317.2683 & 7.1867 & 0.012 & 7.125 & 49047.3949 & 7.1602 & 0.009 & 7.099 \\
\hline 48317.2826 & 7.1824 & 0.013 & 7.121 & 49047.6431 & 7.1776 & 0.010 & 7.116 \\
\hline 48322.9710 & 7.2103 & 0.007 & 7.149 & 49047.6575 & 7.1718 & 0.009 & 7.111 \\
\hline 48323.0455 & 7.1897 & 0.009 & 7.128 & 49047.7320 & 7.1710 & 0.009 & 7.110 \\
\hline 48323.0598 & 7.2171 & 0.008 & 7.156 & 49047.7463 & 7.1712 & 0.010 & 7.110 \\
\hline 48323.3122 & 7.2063 & 0.008 & 7.145 & 49047.8209 & 7.1732 & 0.009 & 7.112 \\
\hline 48323.3265 & 7.2085 & 0.010 & 7.147 & 49047.8352 & 7.1607 & 0.008 & 7.099 \\
\hline 48323.4011 & 7.1930 & 0.007 & 7.132 & 49053.4135 & 7.1785 & 0.008 & 7.117 \\
\hline 48323.4154 & 7.1903 & 0.006 & 7.129 & 49053.4279 & 7.2007 & 0.007 & 7.139 \\
\hline 48323.4900 & 7.2006 & 0.007 & 7.139 & 49053.5024 & 7.1748 & 0.009 & 7.114 \\
\hline 48323.5043 & 7.2062 & 0.009 & 7.145 & 49053.5167 & 7.1760 & 0.011 & 7.115 \\
\hline 48359.4706 & 7.2092 & 0.009 & 7.148 & 49053.5913 & 7.1690 & 0.008 & 7.108 \\
\hline 48393.3316 & 7.2130 & 0.010 & 7.152 & 49053.6056 & 7.1613 & 0.010 & 7.100 \\
\hline 48393.4062 & 7.2248 & 0.007 & 7.164 & 49053.8561 & 7.1615 & 0.007 & 7.100 \\
\hline 48393.4205 & 7.2077 & 0.012 & 7.146 & 49053.8704 & 7.1701 & 0.007 & 7.109 \\
\hline 48393.4951 & 7.2170 & 0.008 & 7.156 & 49053.9450 & 7.1604 & 0.011 & 7.099 \\
\hline 48393.5094 & 7.2208 & 0.009 & 7.160 & 49054.0339 & 7.1688 & 0.009 & 7.108 \\
\hline 48393.5840 & 7.2114 & 0.006 & 7.150 & & & & \\
\hline
\end{tabular}


P. Harmanec et al.: Duplicity of the Be star BR CMi

Table B.5. Individual ASAS-3 $V$ band observations from diaphragm 1 without large errors.

\begin{tabular}{|c|c|c|c|c|c|c|c|c|}
\hline $\begin{array}{c}\text { HJD } \\
-2400000\end{array}$ & $\begin{array}{c}V \\
(\mathrm{mag})\end{array}$ & $\begin{array}{c}\mathrm{rms} \\
(\mathrm{mag})\end{array}$ & $\begin{array}{c}\text { HJD } \\
-2400000\end{array}$ & $\begin{array}{c}V \\
(\mathrm{mag})\end{array}$ & $\begin{array}{c}\mathrm{rms} \\
(\mathrm{mag})\end{array}$ & $\begin{array}{c}\text { HJD } \\
-2400000 \\
\end{array}$ & $\begin{array}{c}V \\
\text { (mag) }\end{array}$ & $\begin{array}{c}\mathrm{rms} \\
(\mathrm{mag})\end{array}$ \\
\hline 52552.8776 & 7.156 & 0.034 & 52970.7859 & 7.118 & 0.037 & 54107.7196 & 7.126 & 0.037 \\
\hline 52557.8567 & 7.145 & 0.036 & 52973.8098 & 7.129 & 0.032 & 54127.7145 & 7.114 & 0.037 \\
\hline 52559.8729 & 7.148 & 0.035 & 52975.7732 & 7.089 & 0.038 & 54141.7008 & 7.144 & 0.038 \\
\hline 52577.8040 & 7.143 & 0.036 & 52979.7768 & 7.147 & 0.033 & 54146.6021 & 7.119 & 0.038 \\
\hline 52623.7460 & 7.175 & 0.038 & 52986.7882 & 7.143 & 0.039 & 54152.5591 & 7.095 & 0.037 \\
\hline 52625.7454 & 7.124 & 0.036 & 52989.7358 & 7.101 & 0.037 & 54154.5939 & 7.160 & 0.037 \\
\hline 52637.7183 & 7.145 & 0.036 & 52991.7752 & 7.155 & 0.038 & 54174.6296 & 7.149 & 0.040 \\
\hline 52641.7108 & 7.132 & 0.036 & 52996.7692 & 7.128 & 0.036 & 54188.6063 & 7.138 & 0.039 \\
\hline 52645.7047 & 7.106 & 0.038 & 53001.7538 & 7.084 & 0.031 & 54201.5468 & 7.113 & 0.038 \\
\hline 52650.6776 & 7.156 & 0.036 & 53006.6958 & 7.099 & 0.034 & 54204.5454 & 7.101 & 0.039 \\
\hline 52652.6885 & 7.104 & 0.036 & 53008.7208 & 7.097 & 0.039 & 54216.5315 & 7.094 & 0.039 \\
\hline 52660.6893 & 7.120 & 0.035 & 53016.7075 & 7.141 & 0.039 & 54233.4825 & 7.115 & 0.037 \\
\hline 52664.6776 & 7.115 & 0.037 & 53018.7030 & 7.140 & 0.040 & 54235.4863 & 7.100 & 0.039 \\
\hline 52666.6750 & 7.114 & 0.040 & 53028.7644 & 7.105 & 0.040 & 54247.4538 & 7.102 & 0.039 \\
\hline 52670.6739 & 7.121 & 0.038 & 53031.6263 & 7.132 & 0.034 & 54365.8936 & 7.105 & 0.036 \\
\hline 52674.6623 & 7.153 & 0.036 & 53035.6532 & 7.137 & 0.040 & 54383.8650 & 7.068 & 0.038 \\
\hline 52676.6485 & 7.148 & 0.037 & 53046.6032 & 7.082 & 0.036 & 54412.8547 & 7.141 & 0.038 \\
\hline 52678.6339 & 7.089 & 0.039 & 53049.6167 & 7.150 & 0.038 & 54436.7788 & 7.100 & 0.039 \\
\hline 52681.6881 & 7.163 & 0.040 & 53054.5926 & 7.099 & 0.035 & 54455.7297 & 7.104 & 0.039 \\
\hline 52691.6242 & 7.104 & 0.039 & 53058.6732 & 7.094 & 0.038 & 54470.6969 & 7.145 & 0.039 \\
\hline 52693.6365 & 7.140 & 0.038 & 53060.6989 & 7.103 & 0.039 & 54479.6571 & 7.085 & 0.040 \\
\hline 52697.6305 & 7.101 & 0.036 & 53068.5635 & 7.144 & 0.040 & 54492.7149 & 7.086 & 0.039 \\
\hline 52699.6208 & 7.132 & 0.039 & 53077.5445 & 7.122 & 0.034 & 54494.7342 & 7.095 & 0.040 \\
\hline 52701.6187 & 7.164 & 0.036 & 53102.5989 & 7.143 & 0.040 & 54537.5394 & 7.123 & 0.040 \\
\hline 52703.6144 & 7.108 & 0.038 & 53107.5350 & 7.144 & 0.040 & 54558.6078 & 7.088 & 0.039 \\
\hline 52707.6045 & 7.169 & 0.037 & 53109.5477 & 7.132 & 0.039 & 54570.4912 & 7.088 & 0.040 \\
\hline 52718.5269 & 7.109 & 0.039 & 53111.5476 & 7.090 & 0.040 & 54601.4685 & 7.134 & 0.039 \\
\hline 52734.5519 & 7.138 & 0.039 & 53113.5671 & 7.140 & 0.040 & 54762.8482 & 7.127 & 0.037 \\
\hline 52740.5074 & 7.158 & 0.039 & 53115.5672 & 7.140 & 0.035 & 54774.8284 & 7.138 & 0.038 \\
\hline 52751.5004 & 7.143 & 0.040 & 53123.4834 & 7.093 & 0.040 & 54778.8182 & 7.075 & 0.038 \\
\hline 52760.5200 & 7.134 & 0.037 & 53125.5273 & 7.120 & 0.038 & 54801.7777 & 7.130 & 0.037 \\
\hline 52786.4622 & 7.134 & 0.038 & 53133.5104 & 7.144 & 0.038 & 54804.7718 & 7.108 & 0.037 \\
\hline 52911.8901 & 7.093 & 0.037 & 53144.4748 & 7.096 & 0.034 & 54807.7563 & 7.145 & 0.036 \\
\hline 52916.8806 & 7.097 & 0.037 & 53291.8729 & 7.082 & 0.040 & 54816.7776 & 7.087 & 0.040 \\
\hline 52926.8593 & 7.153 & 0.037 & 53298.8666 & 7.085 & 0.039 & 54819.7738 & 7.145 & 0.039 \\
\hline 52932.8498 & 7.150 & 0.034 & 53333.8126 & 7.149 & 0.040 & 54822.7171 & 7.091 & 0.039 \\
\hline 52940.8298 & 7.147 & 0.037 & 53357.7288 & 7.114 & 0.038 & 54825.7243 & 7.129 & 0.040 \\
\hline 52943.8327 & 7.099 & 0.039 & 53459.5662 & 7.079 & 0.038 & 54831.7039 & 7.138 & 0.037 \\
\hline 52949.8286 & 7.095 & 0.038 & 53466.5452 & 7.079 & 0.039 & 54834.6914 & 7.111 & 0.036 \\
\hline 52952.8292 & 7.148 & 0.040 & 53469.5526 & 7.129 & 0.038 & 54836.7857 & 7.091 & 0.038 \\
\hline 52955.8312 & 7.084 & 0.036 & 53650.8382 & 7.162 & 0.040 & & & \\
\hline 52963.8062 & 7.104 & 0.037 & 54091.7187 & 7.096 & 0.040 & & & \\
\hline
\end{tabular}


Table B.6. Individual $U B V$ band observations.

\begin{tabular}{|c|c|c|c|c|c|c|c|c|}
\hline $\begin{array}{c}\text { HJD } \\
-2400000\end{array}$ & Weight & $\begin{array}{c}V \\
(\mathrm{mag})\end{array}$ & $\begin{array}{c}B \\
(\mathrm{mag})\end{array}$ & $\begin{array}{c}U \\
\text { (mag) }\end{array}$ & $\begin{array}{c}B-V \\
(\mathrm{mag})\end{array}$ & $\begin{array}{c}U-B \\
(\mathrm{mag}))\end{array}$ & $X$ & $\triangle X$ \\
\hline \multicolumn{9}{|l|}{ Hvar } \\
\hline 55574.4833 & 1.00 & 7.158 & 7.408 & 7.424 & 0.250 & 0.016 & 1.229 & 0.044 \\
\hline 55574.4901 & 1.00 & 7.168 & 7.415 & 7.458 & 0.247 & 0.043 & 1.235 & 0.042 \\
\hline 55574.4990 & 1.00 & 7.160 & 7.410 & 7.432 & 0.250 & 0.022 & 1.246 & 0.039 \\
\hline 55578.4494 & 1.00 & 7.121 & 7.399 & 7.447 & 0.278 & 0.048 & 1.225 & 0.052 \\
\hline 55578.4566 & 1.00 & 7.125 & 7.405 & 7.434 & 0.280 & 0.029 & 1.224 & 0.049 \\
\hline 55578.4621 & 1.00 & 7.124 & 7.397 & 7.436 & 0.273 & 0.039 & 1.225 & 0.047 \\
\hline 55585.4986 & 1.00 & 7.098 & 7.391 & 7.432 & 0.293 & 0.041 & 1.308 & 0.031 \\
\hline 55585.5069 & 1.00 & 7.101 & 7.371 & 7.403 & 0.270 & 0.032 & 1.335 & 0.029 \\
\hline 55585.5152 & 1.00 & 7.106 & 7.390 & 7.435 & 0.284 & 0.045 & 1.366 & 0.027 \\
\hline 55585.5165 & 1.00 & 7.111 & 7.393 & 7.440 & 0.282 & 0.047 & 1.371 & 0.027 \\
\hline 55586.4851 & 1.00 & 7.124 & 7.398 & 7.424 & 0.274 & 0.026 & 1.280 & 0.034 \\
\hline 55586.4948 & 1.00 & 7.131 & 7.399 & 7.433 & 0.268 & 0.034 & 1.306 & 0.032 \\
\hline 55586.5039 & 1.00 & 7.130 & 7.409 & 7.432 & 0.279 & 0.023 & 1.334 & 0.029 \\
\hline 55594.4271 & 1.50 & 7.175 & 7.417 & 7.433 & 0.242 & 0.016 & 1.229 & 0.044 \\
\hline 55594.4356 & 1.50 & 7.182 & 7.417 & 7.430 & 0.235 & 0.013 & 1.235 & 0.041 \\
\hline 55594.4444 & 1.50 & 7.184 & 7.426 & 7.435 & 0.242 & 0.009 & 1.246 & 0.039 \\
\hline 55596.4157 & 1.00 & 7.160 & 7.424 & 7.441 & 0.264 & 0.017 & 1.226 & 0.046 \\
\hline 55596.4243 & 1.00 & 7.161 & 7.426 & 7.437 & 0.265 & 0.011 & 1.230 & 0.043 \\
\hline 55596.4325 & 1.00 & 7.154 & 7.434 & 7.433 & 0.280 & -0.001 & 1.238 & 0.041 \\
\hline 55597.3054 & 1.50 & 7.136 & 7.399 & 7.421 & 0.263 & 0.022 & 1.468 & 0.121 \\
\hline 55597.3134 & 1.50 & 7.133 & 7.397 & 7.440 & 0.264 & 0.043 & 1.425 & 0.111 \\
\hline 55597.3212 & 1.50 & 7.147 & 7.401 & 7.434 & 0.254 & 0.033 & 1.389 & 0.102 \\
\hline 55599.3682 & 0.50 & 7.121 & 7.391 & 7.425 & 0.270 & 0.034 & 1.245 & 0.063 \\
\hline 55599.3724 & 0.50 & 7.129 & 7.400 & 7.444 & 0.271 & 0.044 & 1.239 & 0.061 \\
\hline 55599.3754 & 0.50 & 7.125 & 7.395 & 7.431 & 0.270 & 0.036 & 1.236 & 0.059 \\
\hline 55645.3479 & 0.50 & 7.152 & 7.406 & 7.437 & 0.254 & 0.031 & 1.365 & 0.028 \\
\hline 55645.3561 & 0.50 & 7.156 & 7.412 & 7.436 & 0.256 & 0.024 & 1.400 & 0.026 \\
\hline 55645.3604 & 0.50 & 7.167 & 7.418 & 7.452 & 0.251 & 0.034 & 1.421 & 0.025 \\
\hline 55652.3121 & 0.50 & 7.147 & 7.392 & 7.409 & 0.245 & 0.017 & 1.310 & 0.031 \\
\hline 55652.3220 & 0.50 & 7.167 & 7.428 & 7.454 & 0.261 & 0.026 & 1.342 & 0.029 \\
\hline 55652.3250 & 0.50 & 7.174 & 7.419 & 7.449 & 0.245 & 0.030 & 1.353 & 0.028 \\
\hline 55654.3169 & 1.00 & 7.160 & 7.409 & 7.451 & 0.249 & 0.042 & 1.344 & 0.029 \\
\hline 55654.3253 & 1.00 & 7.163 & 7.410 & 7.434 & 0.247 & 0.024 & 1.377 & 0.027 \\
\hline 55654.3333 & 1.00 & 7.161 & 7.411 & 7.428 & 0.250 & 0.017 & 1.413 & 0.025 \\
\hline 55655.3064 & 1.00 & 7.136 & 7.405 & 7.440 & 0.269 & 0.035 & 1.319 & 0.031 \\
\hline 5565 & 1.00 & 7.131 & 7.404 & 7.443 & 0.273 & 0.039 & 1.347 & 0.029 \\
\hline 55655.3230 & 1.00 & 7.123 & 7.396 & 7.430 & 0.273 & 0.034 & 1.379 & 0.027 \\
\hline 55657.3373 & 1.00 & 7.124 & 7.401 & 7.434 & 0.277 & 0.033 & 1.479 & 0.023 \\
\hline 55657.3423 & 1.00 & 7.128 & 7.418 & 7.480 & 0.290 & 0.062 & 1.510 & 0.021 \\
\hline 55657.3452 & 1.00 & 7.116 & 7.415 & 7.486 & 0.299 & 0.071 & 1.529 & 0.021 \\
\hline 55658.3436 & 0.50 & 7.173 & 7.424 & 7.458 & 0.251 & 0.034 & 1.537 & 0.021 \\
\hline 55658.3477 & 0.50 & 7.165 & 7.419 & 7.454 & 0.254 & 0.035 & 1.566 & 0.020 \\
\hline 55658.3530 & 0.50 & 7.167 & 7.412 & 7.433 & 0.245 & 0.021 & 1.607 & 0.019 \\
\hline 55660.3295 & 1.00 & 7.179 & 7.420 & 7.436 & 0.241 & 0.016 & 1.483 & 0.022 \\
\hline 55660.3307 & 1.00 & 7.185 & 7.421 & 7.437 & 0.236 & 0.016 & 1.490 & 0.022 \\
\hline 55660.3349 & 1.00 & 7.190 & 7.421 & 7.446 & 0.231 & 0.025 & 1.517 & 0.021 \\
\hline 55661.3187 & 1.00 & 7.165 & 7.410 & 7.439 & 0.245 & 0.029 & 1.439 & 0.024 \\
\hline 55661.3273 & 1.00 & 7.160 & 7.410 & 7.455 & 0.250 & 0.045 & 1.487 & 0.022 \\
\hline 55661.3359 & 1.00 & 7.154 & 7.409 & 7.454 & 0.255 & 0.045 & 1.543 & 0.020 \\
\hline 55662.3278 & 0.50 & 7.123 & 7.378 & 7.408 & 0.255 & 0.030 & 1.507 & 0.022 \\
\hline 55662.3407 & 0.50 & 7.108 & 7.363 & 7.395 & 0.255 & 0.032 & 1.599 & 0.019 \\
\hline 55662.3420 & 0.50 & 7.116 & 7.371 & 7.420 & 0.255 & 0.049 & 1.609 & 0.019 \\
\hline 55848.5816 & 1.00 & 7.145 & 7.415 & 7.480 & 0.270 & 0.065 & 1.690 & 0.175 \\
\hline 55848.5922 & 1.00 & 7.145 & 7.422 & 7.455 & 0.277 & 0.033 & 1.599 & 0.152 \\
\hline 55848.5979 & 1.00 & 7.118 & 7.384 & 7.394 & 0.266 & 0.010 & 1.556 & 0.142 \\
\hline 55848.6654 & 1.00 & 7.143 & 7.416 & 7.458 & 0.273 & 0.042 & 1.269 & 0.071 \\
\hline 55850.5676 & 0.50 & 7.097 & 7.369 & 7.406 & 0.272 & 0.037 & 1.779 & 0.198 \\
\hline 55850.5774 & 0.50 & 7.113 & 7.385 & 7.413 & 0.272 & 0.028 & 1.680 & 0.172 \\
\hline 55850.5863 & 0.50 & 7.119 & 7.390 & 7.440 & 0.271 & 0.050 & 1.604 & 0.154 \\
\hline 55851.5815 & 1.00 & 7.145 & 7.408 & 7.428 & 0.263 & 0.020 & 1.622 & 0.158 \\
\hline 55851.5899 & 1.00 & 7.149 & 7.413 & 7.442 & 0.264 & 0.029 & 1.557 & 0.142 \\
\hline 55851.5980 & 1.00 & 7.156 & 7.413 & 7.444 & 0.257 & 0.031 & 1.502 & 0.129 \\
\hline
\end{tabular}


P. Harmanec et al.: Duplicity of the Be star BR CMi

Table B.6. continued.

\begin{tabular}{|c|c|c|c|c|c|c|c|c|}
\hline $\begin{array}{c}\text { HJD } \\
-2400000\end{array}$ & Weight & $\begin{array}{c}V \\
(\mathrm{mag})\end{array}$ & $\begin{array}{c}B \\
(\mathrm{mag})\end{array}$ & $\begin{array}{c}U \\
(\mathrm{mag})\end{array}$ & $\begin{array}{l}B-V \\
(\mathrm{mag})\end{array}$ & $\begin{array}{c}U-B \\
(\mathrm{mag}))\end{array}$ & $X$ & $\triangle X$ \\
\hline 55852.6273 & 1.50 & 7.179 & 7.429 & 7.444 & 0.250 & 0.015 & 1.347 & 0.092 \\
\hline 55852.6355 & 1.50 & 7.175 & 7.423 & 7.443 & 0.248 & 0.020 & 1.319 & 0.085 \\
\hline 55852.6445 & 1.50 & 7.178 & 7.423 & 7.441 & 0.245 & 0.018 & 1.294 & 0.078 \\
\hline 55853.6267 & 1.00 & 7.184 & 7.422 & 7.426 & 0.238 & 0.004 & 1.340 & 0.090 \\
\hline 55853.6346 & 1.00 & 7.177 & 7.422 & 7.438 & & 0.016 & 1.314 & 0.083 \\
\hline 55853.6426 & 1.00 & 7.185 & 7.431 & 7.442 & 0.246 & 0.011 & 1.292 & 0.077 \\
\hline 55858.6678 & 1.50 & 7.161 & 7.415 & 7.440 & 0.254 & 0.025 & 1.230 & 0.056 \\
\hline 55858.6757 & 1.50 & 7.155 & 7.413 & 7.437 & 0.258 & 0.024 & 1.226 & 0.053 \\
\hline 55879.5943 & 1.50 & 7.182 & 7.424 & 7.435 & 0.242 & 0.011 & 1.250 & 0.065 \\
\hline 55879.6022 & 1.50 & 7.183 & 7.436 & 7.444 & 0.253 & 0.008 & 1.240 & 0.061 \\
\hline 55879.6098 & 1.50 & 7.190 & 7.423 & 7.434 & 0.233 & 0.011 & 1.232 & 0.057 \\
\hline 55881.6494 & 1.50 & 7.121 & 7.393 & 7.423 & 0.272 & 0.030 & 1.238 & 0.041 \\
\hline 55881.6545 & 1.50 & 7.115 & 7.394 & 7.426 & 0.279 & 0.032 & 1.245 & 0.039 \\
\hline 55881.6579 & 1.50 & 7.118 & 7.401 & 7.432 & 0.283 & 0.031 & 1.250 & 0.038 \\
\hline 55938.4426 & 1.50 & 7.171 & 7.420 & 7.437 & 0.249 & 0.017 & 1.242 & 0.062 \\
\hline 55938.4505 & 1.50 & 7.175 & 7.418 & 7.430 & 0.243 & 0.012 & 1.233 & 0.058 \\
\hline 55938.4552 & 1.50 & 7.176 & 7.420 & 7.436 & & 0.016 & 1.229 & 0.056 \\
\hline 55939.3829 & 1.50 & 7.133 & 7.402 & 7.430 & & 0.028 & 1.398 & 0.104 \\
\hline 55939.3882 & 1.50 & 7.134 & 7.408 & 7.433 & & 0.025 & 1.375 & 0.099 \\
\hline 55939.3916 & 1.50 & 7.136 & 7.403 & 7.430 & 0.267 & 0.027 & 1.361 & 0.095 \\
\hline 55942.4525 & 1.50 & 7.153 & 7.409 & 7.430 & 0.256 & 0.021 & 1.225 & 0.052 \\
\hline 55942.4592 & 1.50 & 7.160 & 7.413 & 7.436 & 0.253 & 0.023 & 1.224 & 0.050 \\
\hline 55942.4674 & 1.50 & 7.161 & 7.411 & 7.434 & 0.250 & 0.023 & 1.225 & 0.047 \\
\hline 55943.4365 & 1.50 & 7.177 & 7.413 & 7.427 & 0.236 & 0.014 & 1.234 & 0.058 \\
\hline 55943.4449 & 1.50 & 7.179 & 7.420 & 7.436 & 0.241 & 0.016 & 1.227 & 0.054 \\
\hline 55943.4499 & 1.50 & 7.178 & 7.418 & 7.431 & 0.240 & 0.013 & 1.225 & 0.052 \\
\hline 55945.3602 & 1.50 & 7.157 & 7.409 & 7.439 & 0.252 & 0.030 & 1.429 & 0.111 \\
\hline 55945.3665 & 1.50 & 7.164 & 7.413 & 7.437 & 0.249 & 0.024 & 1.398 & 0.104 \\
\hline 55945.3698 & 1.50 & 7.168 & 7.415 & 7.431 & 0.247 & 0.016 & 1.384 & 0.101 \\
\hline 56001.3240 & 1.50 & 7.177 & 7.421 & 7.430 & 0.2 & 0.009 & 1.241 & 0.040 \\
\hline 56001.3316 & 1.50 & 7.173 & 7.415 & 7.426 & 0.242 & 0.011 & 1.251 & 0.038 \\
\hline 56001.3366 & 1.50 & 7.175 & 7.419 & 7.430 & & 0.011 & 1.260 & 0.037 \\
\hline 02.3518 & 1.50 & 7.184 & 7.418 & 7.435 & 0.234 & 0.017 & 1.301 & 0.032 \\
\hline 56002.3596 & 1.50 & 7.176 & 7.415 & 7.434 & 0.239 & 0.019 & 1.324 & 0.030 \\
\hline 56002.3643 & 1.50 & 7.186 & 7.435 & 7.452 & 0.249 & 0.017 & 1.340 & 0.029 \\
\hline 56013.3093 & 0.50 & 7.142 & 7.408 & 7.436 & 0.266 & 0.028 & 1.273 & 0.035 \\
\hline 56013.3142 & 0.50 & 7.148 & 7.409 & 7.431 & 0.261 & 0.022 & 1.284 & 0.034 \\
\hline 56013.3203 & 0.50 & 7.146 & 7.396 & 7.424 & 0.250 & 0.028 & 1.299 & 0.032 \\
\hline 56015.3123 & 0.50 & 7.181 & 7.432 & 7.443 & 0.25 & 0.011 & 1.293 & 0.033 \\
\hline 56015.3185 & 0.50 & .184 & 7.439 & 7.475 & 0.255 & 0.036 & 1.310 & 0.031 \\
\hline 56015.3245 & 0.50 & 7.192 & 7.435 & 7.446 & 0.243 & 0.011 & 1.329 & 0.030 \\
\hline \multicolumn{9}{|l|}{ SAAO } \\
\hline 55567.4069 & 1.00 & 7.136 & 7.417 & 7.431 & 0.281 & 0.014 & 1.453 & 0.002 \\
\hline 55567.4146 & 1.00 & 7.139 & 7.425 & 7.429 & 0.286 & 0.004 & 1.420 & 0.002 \\
\hline 55567.4217 & 1.00 & 7.144 & 7.420 & 7.427 & 0.276 & 0.007 & 1.394 & 0.002 \\
\hline 55570.5248 & 0.50 & 7.175 & 7.434 & 7.425 & 0.259 & -0.009 & 1.415 & 0.004 \\
\hline 55573.4850 & 1.00 & 7.144 & 7.409 & 7.415 & 0.265 & 0.006 & 1.331 & 0.003 \\
\hline 55573.4914 & 1.00 & 7.122 & 7.394 & 7.426 & 0.272 & 0.032 & 1.343 & 0.003 \\
\hline 55573.4981 & 1.00 & 7.119 & 7.400 & 7.415 & 0.281 & 0.015 & 1.357 & 0.003 \\
\hline 55574.5248 & 1.00 & 7.180 & 7.428 & 7.462 & 0.248 & 0.034 & 1.461 & 0.004 \\
\hline 55574.5308 & 1.00 & 7.154 & 7.409 & 7.426 & 0.255 & 0.017 & 1.491 & 0.004 \\
\hline 55574.5371 & 1.00 & 7.166 & 7.403 & 7.421 & 0.237 & 0.018 & 1.526 & 0.005 \\
\hline 55575.5287 & 1.00 & 7.180 & 7.421 & 7.433 & 0.241 & 0.012 & 1.494 & 0.005 \\
\hline 55575.5345 & 1.00 & 7.169 & 7.429 & 7.415 & 0.260 & -0.014 & 1.528 & 0.005 \\
\hline 55575.5408 & 1.00 & 7.191 & 7.440 & 7.426 & 0.249 & -0.014 & 1.568 & 0.005 \\
\hline 55577.5457 & 1.50 & 7.135 & 7.415 & 7.412 & 0.280 & -0.003 & 1.646 & 0.006 \\
\hline 55578.5443 & 0.50 & 7.130 & 7.401 & 7.420 & 0.271 & 0.019 & 1.657 & 0.006 \\
\hline 55578.5505 & 0.50 & 7.133 & 7.420 & 7.436 & 0.287 & 0.016 & 1.712 & 0.006 \\
\hline 55579.5226 & 1.00 & 7.110 & 7.407 & 7.431 & 0.297 & 0.024 & 1.522 & 0.005 \\
\hline 55579.5288 & 1.00 & 7.125 & 7.401 & 7.418 & 0.276 & 0.017 & 1.560 & 0.005 \\
\hline 55579.5345 & 1.00 & 7.118 & 7.382 & 7.422 & 0.264 & 0.040 & 1.601 & 0.005 \\
\hline
\end{tabular}

